Japan. J. Math.

Vol. 21, No. 2, 1995

\title{
Examples of canonical decompositions of hyperbolic link complements
}

\author{
By Makoto SAKuma and Jeffrey WeEks
}

(Received May 11, 1994)

For each cusped hyperbolic manifold $M$, there is a canonical way of decomposing $M$ into ideal cells, which we call the canonical decomposition of $M$ (see [EP, $\mathrm{W}]$ ). If we could determine the combinatorial structures of the canonical decompositions, then we can solve the homeomorphism problems of these manifolds and can also calculate the mapping class groups. In fact, by virtue of the Mostow rigidity theorem, two such manifolds of dimension greater than 2 are homeomorphic if and only if their canonical decompositions have the same combinatorial structures, and their mapping class groups are isomorphic to the combinatorial automorphism groups of their canonical decompositions.

The canonical decompositions are the geometric duals of the Ford domains (see [EP, Section 4]). In the pioneering works [R2, 3, 4], Riley constructed the Ford domains of several knot complements and computed their symmetry groups. In the lecture note of Thurston [Th], we can find beautiful constructions of the hyperbolic structures on the complements of some series of symmetric links as the unions of two ideal polyhedra; most of these decompositions are in fact canonical. These constructions were generalized by Hatcher $[\mathrm{H}]$, where he found the Ford domains for several arithmetic link complements (see also [AR1]). J $\phi$ rgensen [Jr] constructed the canonical decompositions of the once-punctured torus bundles over a circle; their combinatorial structures are nicely explained in $[\mathrm{FH}]$.

The computer program SnapPea developed by the second-named author determines the canonical decompositions of cusped hyperbolic 3-manifolds, and it quickly determines whether two such manifolds are isometric and computes the isometry groups (cf. [W]). In particular, SnapPea can decide whether two hyperbolic links are equivalent and can compute their symmetry groups (see [AHW, HW] and compare with $[\mathrm{KS}]$ ). For example, it quickly tells us that the mutually mutant knots, the Kinoshita-Terasaka and the Conway knots, are inequivalent by showing that the canonical decompositions of their complements consist of 12 and 14 ideal tetrahedra respectively; further it shows that their symmetry groups are trivial. It seems to be very difficult to obtain these results by appealing to algebraic invariants. (See [BS] for nice topological proofs of these results.) Thus SnapPea is a magic 
wand for practical knot theorists. However, there remain the following problems:

(1) Though SnapPea gives us the combinatorial data of the canonical decompositions, we cannot see the canonical decompositions. How can we see and understand them?

(2) SnapPea does not tell us explicit information concerning a family consisting of infinitely many knots, e.g., the family of 2-bridge knots. How can we find the canonical decompositions of their complements simultaneously?

This paper is a progress report of our attempt to study these problems. In Chapter I, we recall the construction of the canonical decomposition through the convex hull construction [EP, W] and show that the decompositions of the complements of certain family of unusually symmetric links given by [Th] are canonical. In Chapter II, we construct certain topological ideal triangulations of the 2-bridge link complements imitating J $\phi$ rgensen's construction described in $[\mathrm{FH}]$. We conjecture that these are the canonical decompositions; in fact, we have checked this conjecture for the 2-bridge links with $\leq 10$ crossings by using SnapPea. We also determine the hyperbolicity equations for these decompositions and discuss their relations to Riley's work [R1] on parabolic representations of 2-bridge knot groups. In Chapter III, we present a few conjectures related to the alternating links and unknotting tunnels, which arose from our experiments.

\section{Chapter I. Basic constructions}

\section{I.1. Convex hull construction}

The Minkowski space $\mathbb{E}^{n, 1}$ is the real vector space $\mathbb{R}^{n+1}$ with the inner product $\langle\mathbf{x}, \mathbf{y}\rangle=-x_{0} y_{0}+x_{1} y_{1}+\cdots+x_{n} y_{n}$. The set $\left\{\mathbf{x} \in \mathbb{E}^{n, 1} \mid\langle\mathbf{x}, \mathbf{x}\rangle=-1, x_{0}>0\right\}$ forms the Minkowski space model for the hyperbolic $n$-space $\mathbb{H}^{n}$. The radial projection from $(-1,0, \ldots, 0)$ to the unit disk in the plane $x_{0}=0$ gives an isometry of the Minkowski space model with the Poincaré disk model, and the radial projection from the origin of $\mathbb{E}^{n, 1}$ to the unit disk in the plane $x_{0}=1$ with center $(1,0, \ldots, 0)$ gives an isometry of the Minkowski model with the Klein model.

Let $M$ be a cusped hyperbolic manifold, and choose horospherical cross sections of the cusps bounding equal volumes. The preimages of the cusp cross sections in the universal cover $\mathbb{H}^{n}$ form an infinite set, say $S$, of horospheres, invariant under the action of the covering transformation group. In the Minkowski space model, each horosphere is the intersection of $\mathbb{H}^{n}$ with a hyperplane, say $W$, whose normal vector is lightlike; we associate to each horosphere the unique lightlike vector $\mathbf{v}$ such that $\langle\mathbf{v}, \mathbf{w}\rangle=-1$ for all $\mathbf{w} \in W$. Let $V$ be the set of points on the light cone corresponding to the horospheres in $S$. Then $V$ is invariant under the action of the covering transformation group. Let $C$ be the closed convex hull of $V$ in $\mathbb{E}^{n, 1}$. Then $\partial C$ is the union of a set of rays $\{t \mathbf{v} \mid t \geq 1, \mathbf{v} \in V\}$ on the light-cone and a countable number of $n$-dimensional faces; each $n$-dimensional face is the convex 
hull of a finite number of points in $V$, and the set of the $n$-dimensional faces is locally finite in the interior of the light-cone [EP, Lemma 3.3 and Proposition 3.5]. Projecting the faces of $\partial C$ radially from the origin of $\mathbb{E}^{n, 1}$ onto $\mathbb{H}^{n}$, we obtain an ideal cell decomposition of $\mathbb{H}^{n}$. This is invariant under the covering transformation group and projects down to an ideal cell decomposition of $M$. This decomposition does not depend on the initial choice of cusp cross sections, and it is called the canonical decomposition of $M$. The same construction works even if we choose cusp cross sections bounding not necessarily equal volumes, and the resulting ideal cell decomposition (which depends only on the ratios of the volumes bounded by the chosen cusp cross sections) is called a Euclidean decomposition of $M$ [EP].

Next, we give a condition for a given ideal cell decomposition $\mathcal{D}$ of $M$ to be canonical. Let $\sigma$ be an $n$-cell in $\mathcal{D}$ and $\widetilde{\sigma}$ a lift of $\sigma$ to $\mathbb{H}^{n}$. Let $\mathbf{v}_{1}, \ldots, \mathbf{v}_{k}$ be the points in $V$ corresponding to the ideal vertices of $\widetilde{\sigma}$, and let $\widehat{\sigma}$ be the convex hull of $\left\{\mathbf{v}_{1}, \ldots, \mathbf{v}_{k}\right\}$. Then the following condition is necessary for $\mathcal{D}$ to be canonical (see [EP, Proposition 3.5]):

(I.1.1) The affine hull $A(\widehat{\sigma})$ of $\widehat{\sigma}$ is a Euclidean $n$-space in $\mathbb{E}^{n, 1}$; i.e., the restriction of the Minkowski metric to $A(\widehat{\sigma})$ is positive definite.

In the following, we assume (I.1.1) is satisfied and call $\widehat{\sigma}$ the Euclidean cell corresponding to $\widetilde{\sigma}$. Let $\tau$ be an $(n-1)$-cell of $\mathcal{D}$, and let $\sigma_{1}$ and $\sigma_{2}$ be the $n$-cells of $\mathcal{D}$ incident to $\tau$. Let $\widetilde{\sigma}_{1}, \widetilde{\sigma}_{2}$, and $\widetilde{\tau}$, respectively, be lifts of $\sigma_{1}, \sigma_{2}$, and $\tau$, such that $\widetilde{\sigma}_{1} \cap \widetilde{\sigma}_{2}=\widetilde{\tau}$. Let $\widehat{\sigma}_{1}, \widehat{\sigma}_{2}$, and $\widehat{\tau}$, respectively, be the Euclidean cells in $\mathbb{E}^{n, 1}$ corresponding to $\widetilde{\sigma}_{1}, \widetilde{\sigma}_{2}$, and $\widetilde{\tau}$. Then we need the following condition:

(I.1.2) The dihedral angle between $\widehat{\sigma}_{1}$ and $\widehat{\sigma}_{2}$ is strictly convex.

This condition guarantees that the set of the $n$-cells of $\mathcal{D}$ is equal that of the canonical decomposition of $M$, so $\mathcal{D}$ is a subdivision of the canonical decomposition. Thus, to conclude that $\mathcal{D}$ is canonical, we further need the following condition:

(I.1.3) $\mathcal{D}$ is the coarsest ideal cell decompositions among the ideal cell decompositions of $M$ having the same $n$-cells.

For a general method checking the condition (I.1.2) using the concept of the "tilt", please see [We, SW]. We present here a criterion that is useful for constructing the canonical decompositions of unusually symmetric hyperbolic manifolds. Suppose (I.1.1) is satisfied. Then we can define the center $o(\widetilde{\sigma})$ of $\widetilde{\sigma}$ as follows: Let $\mathbf{p}$ be the unit normal to $A(\widehat{\sigma})$ in $\mathbb{E}^{n, 1}$; i.e., $\langle\mathbf{p}, \mathbf{p}\rangle=-1$ and $\langle\mathbf{p}, \mathbf{x}-\mathbf{y}\rangle=0$ for any $\mathbf{x}, \mathbf{y} \in A(\widehat{\sigma})$. Then $o(\widetilde{\sigma})=\mathbf{p} \in \mathbb{H}^{n}$. It should be noted that $o(\widetilde{\sigma})$ is not necessarily contained in $\tilde{\sigma}$. If we choose a coordinate of $\mathbb{E}^{n, 1}$ so that $\widehat{\sigma}$ lies in a horizontal plane $x_{0}=$ constant, then $\mathbf{p}=(1,0, \ldots, 0)$. From this fact, we can see that $o(\widetilde{\sigma})$ is characterized by the property that its hyperbolic distance from the horospheres corresponding to the light-like vectors $\mathbf{v}_{1}, \ldots, \mathbf{v}_{k}$ are equal. Here $\mathbf{v}_{1}, \ldots, \mathbf{v}_{k}$ are as in the previous paragraph. This means that $o(\widetilde{\sigma})$ corresponds to a vertex of the Ford domain in case $\mathcal{D}$ is the canonical decomposition [EP, Section 4]. 
Proposition I.1.4. Suppose $\mathcal{D}$ satisfies the conditions (I.1.1) and (I.1.3), and suppose $o(\widetilde{\sigma})$ lies in the interior of $\widetilde{\sigma}$ for any $n$-cell $\sigma$ of $\mathcal{D}$. Then $\mathcal{D}$ is the canonical decomposition of $M$.

Proof. Though this immediately follows from [SW, Theorem 3.1], we give a direct proof here. Let $\widehat{\sigma}_{1}, \widehat{\sigma}_{2}$, and $\widehat{\tau}$ be as in (I.1.2). After a coordinate change we may assume $\widehat{\sigma}_{1}$ lies in the horizontal plane $x_{0}=s_{0}$, where $s_{0}$ is a positive constant. By the assumption, we see $\widehat{\sigma}_{1}$ intersects the $x_{0}$-axis; i.e. $\left(s_{0}, 0, \ldots, 0\right) \in \widehat{\sigma}_{1}$. Suppose (I.1.2) is not satisfied. Then there is a vertex of $\widehat{\sigma}_{2}$ whose $x_{0}$-coordinate is less than or equal to $s_{0}$. Perform a further coordinate change by a rotation about the $x_{0^{-}}$ axis, so that $\widehat{\sigma}_{1}$ and $\widehat{\sigma}_{2}$, respectively, lies in the half spaces $x_{0} / x_{1} \geq s_{0} / s_{1}$ and $x_{0} / x_{1} \leq s_{0} / s_{1}$ for some positive number $s_{1}$ such that $s_{1}<s_{0}$. (see Figure I.1.1).

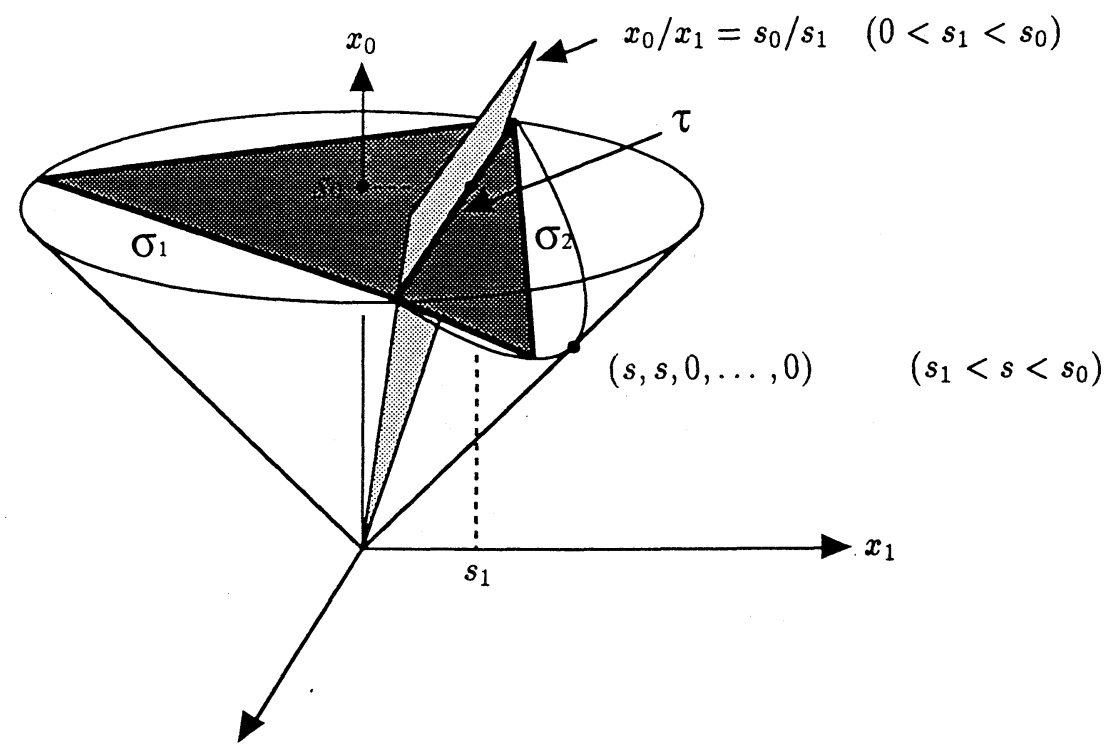

Figure I.1.1

Then there is a positive number $s\left(s_{1}<s<s_{0}\right)$ such that the light-like vector $(s, s, 0, \ldots, 0)$ lies in the affine hull, say $A_{2}$, of $\widehat{\sigma}_{2}$. Let $\left(t_{0}, t_{1}, \ldots, t_{n}\right)$ be the coordinate of $o\left(\widetilde{\sigma}_{2}\right)$. Since $\left(s_{0}, s_{1}, 0, \ldots, 0\right)$ and $(s, s, 0, \ldots, 0)$ lies in $A_{2}$, we have $-t_{0} s_{0}+t_{1} s_{1}=-t_{0} s+t_{1} s$. Hence $t_{1} / t_{0}=\left(s_{1}-s\right) /\left(s_{0}-s\right)<0<s_{1} / s_{0}$. This implies that $o\left(\widetilde{\sigma}_{2}\right) \notin \widetilde{\sigma}_{2}$, a contradiction.

REMARK I.1.5. It should be noted that the converse to the above proposition does not hold. In fact, the canonical decompositions of almost all hyperbolic knots up to 10 crossings (see $[\mathrm{AHW}]$ ) do not satisfy the assumption of the above 
proposition.

\section{I.2. Some symmetric links}

First, we describe the construction of the hyperbolic structures of certain family of link complements due to Thurston [Th, Chapter 6]. To do this, we recall the construction of alternating link complements due to [Me, Ta2, AR1, 2, P] following the explanation by $[\mathrm{AR} 1,2]$. Let $K$ be a prime unsplittable alternating link, and denote a reduced alternating diagram representing $K$ by the same symbol $K$. We regard the diagram $K$ as a 4 -valent graph on the boundary of the 3 -ball $B^{3}$. Then it determines a combinatorial polyhedron, which we denote by the symbol $B_{K}$. Next, two-color the regions of $\partial B_{K}$ checker-board fashion using white and black as illustrated in Figure I.2.1, and assign ' + ' and '-' to the white and black regions respectively.

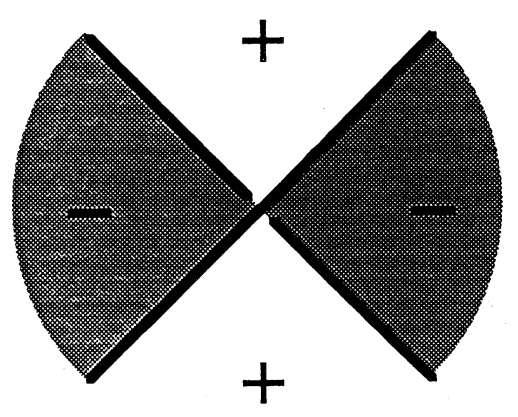

Figure I.2.1

Denote the resulting combinatorial polyhedron by $B_{K}^{+}$. We denote the combinatorial polyhedron obtained from $B_{K}^{+}$by reversing the colors and signs by $B_{K}^{-}$. Each face $F_{i}$ of $B_{K}^{ \pm}$is a combinatorial $n_{i}$-gon, with sign allocation $\sigma_{i}$, and we identify $F_{i}$ with the corresponding face $F_{i}^{\prime}$ of $B_{K}^{\mp}$ by a rotation of $\sigma_{i} \frac{2 \pi}{n_{i}}$, where ' + ' sign denotes clockwise. Denote the resulting topological space by $\bar{M}_{K}$, and let $M_{K}$ denote $\bar{M}_{K}$ with deleted vertices. Then the following holds (see [AR1, Theorem 2.1]):

Proposition I.2.1. $M_{K}$ is canonically homeomorphic to $S^{3}-K$. Each edge of $M_{K}$ corresponds to a crossing of the diagram $K$.

EXAMPLE I.2.2. For each positive integer $n$, let $K(n)$ be the link represented by the braid $\left(\sigma_{1} \sigma_{2}^{-1}\right)^{n}$ (see Figure I.2.2 (a)). This link is considered by [Th, 6.8.11] and is the simplest alternating link obtained from the "basic polyhedron" $(2 \times n)^{*}$ (cf. [C]). This also belongs to the family of knots considered by [KS, Example 1.11]; in fact, $K(n)=S_{n}(10,3)$ in its notation. If $n \geq 2, S^{3}-K(n)$ is obtained by gluing two combinatorial polyhedra as illustrated in Figure I.2.2 (b) (cf. [Th, 6.8.11]). 


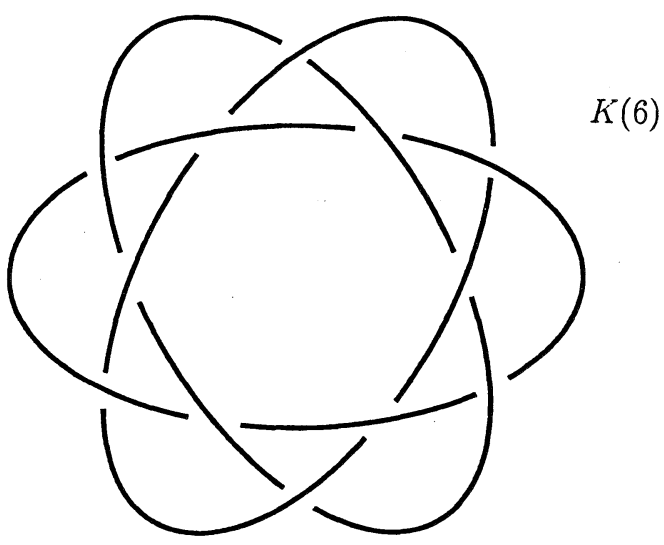

(a)
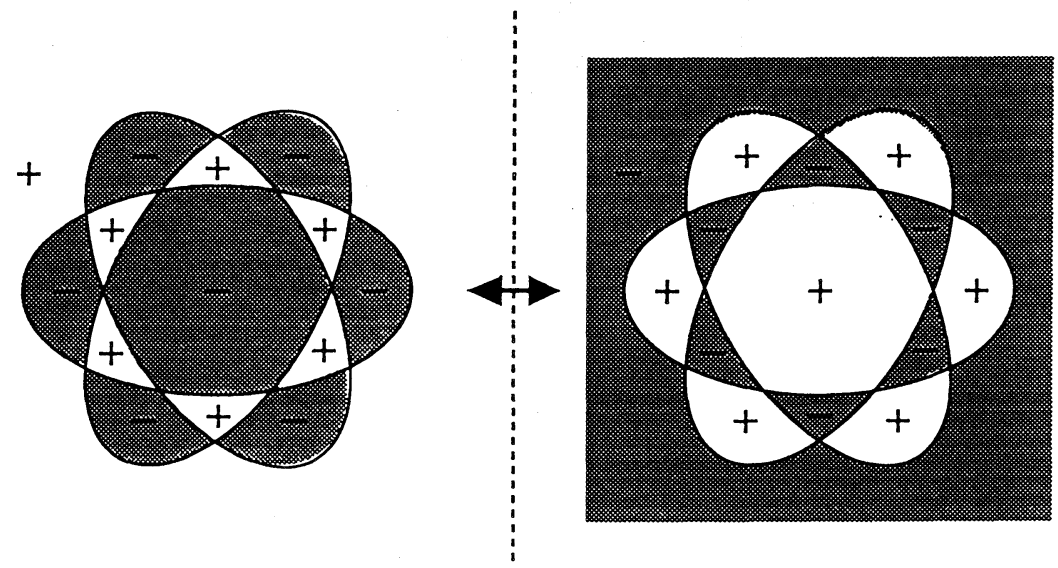

(b)

Figure I.2.2

EXAMPle I.2.3. For each positive integer $n$, let $C(n)$ be the $n$-component chain (see Figure I.2.3 (a)). If $n \geq 3, S^{3}-C(n)$ is obtained from the data illustrated in Figure I.2.3 (b). By shrinking each bigon to an edge, we obtain Figure I.2.3 (c) (cf. [Th, 6.8.1]). 


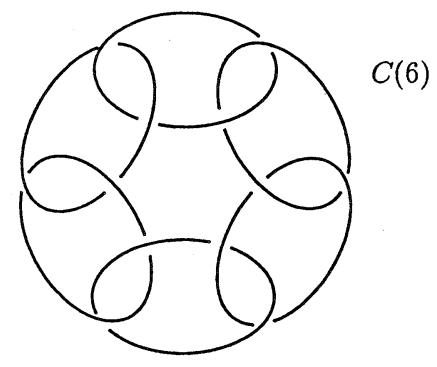

(a)

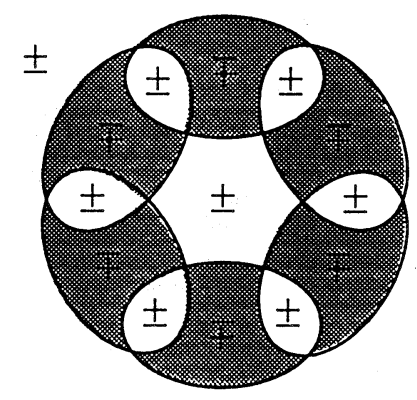

(b)

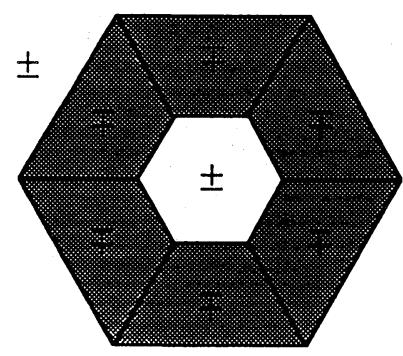

(c)

Figure I.2.3

ExAmPle I.2.4. For each even integer $n \geq 4$, let $C^{\prime}(n)$ be the link as illustrated in Figure I.2.4 (a). Then we see $S^{3}-C^{\prime}(n)$ is obtained by gluing two combinatorial polyhedra as illustrated in Figure I.2.4 (c) (cf. [Th, 6.8.5]).

Let $K$ be one of the links $K(n), C(n)$, and $C^{\prime}(n)$ with $n \geq 3$. In the following, we construct the complete hyperbolic structure on $S^{3}-K$ and determine its canonical decomposition. Let $P(n)$ be the ideal hyperbolic polyhedra as illustrated in Figure I.2.5 (a) or (b) according as $K=K(n)$ or $K \in\left\{C(n), C^{\prime}(n)\right\}$. Here we use the Klein model, and each face is regular with respect to both the hyperbolic metric and the Euclidean metric. 


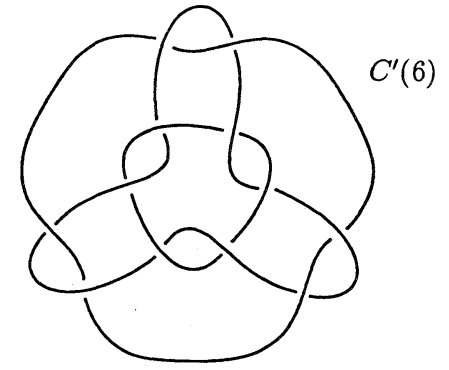

(a)

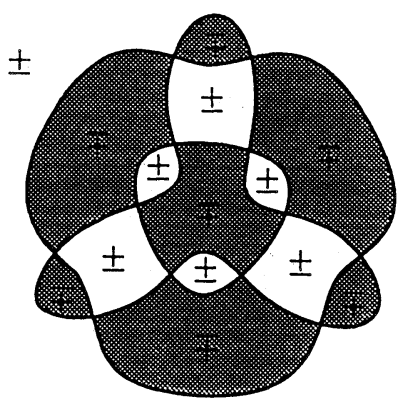

(b)

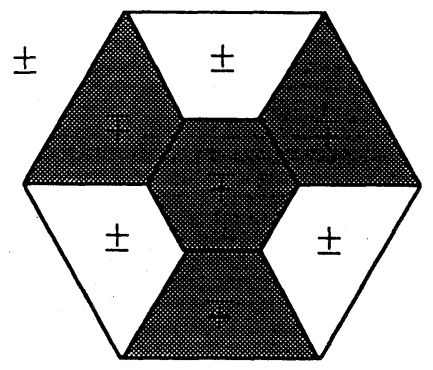

(c)

Figure I.2.4

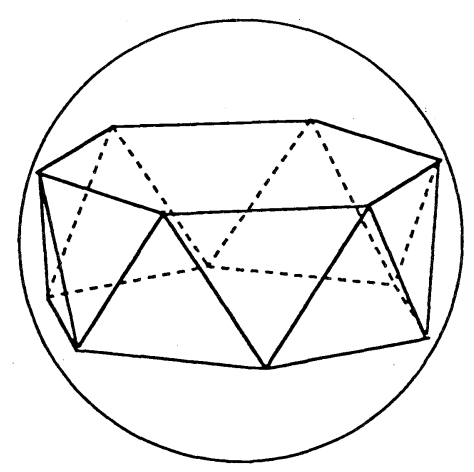

(a)

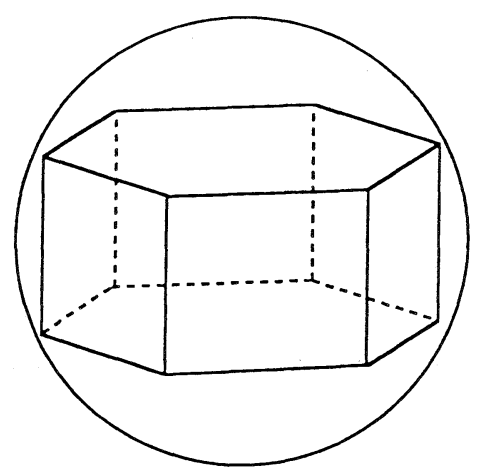

(b)

Figure I.2.5 
By Examples I.2.2-I.2.4, $S^{3}-K$ is obtained from two copies of $P(n)$ by gluing their faces suitably. Since each face is regular, the gluing maps can be chosen to be hyperbolic isometries. Choose $2 n$ horospheres around the vertices of $P(n)$ of the same Euclidean magnitudes in the Klein model. Then the gluing isometries respect the horospherical cross sections. Thus to see that these ideal polyhedra together with the gluing isometries to give the complete hyperbolic structure of the link complement, we have only to check that the total angle around each identified edge is $2 \pi$. But, this can be done by checking that the link of each edge in the cusp cross section is as illustrated in Figure I.2.6 (a) or (b) according as $K=K(n)$ or $K \in\left\{C(n), C^{\prime}(n)\right\}$. (See [Th, Chapter 6.8].)

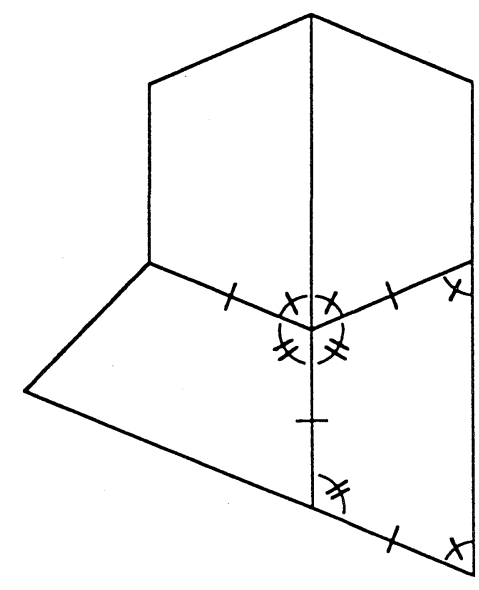

(a)

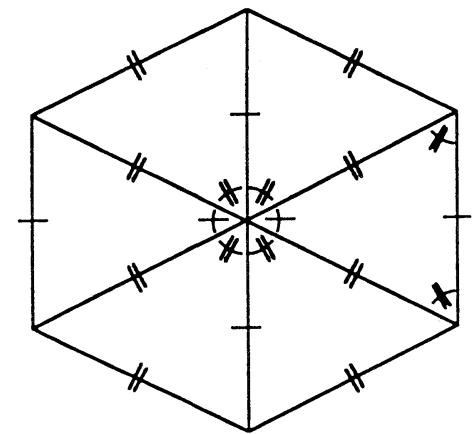

(b)

Figure I.2.6

Moreover, we obtain the following:

Proposition I.2.5. Suppose $K=K(n)$ or $K=C(n)$. Then;

(1) the above decomposition of $S^{3}-K$ into two ideal polyhedra is the canonical decomposition, and

(2) $\operatorname{Isom}\left(S^{3}-K\right)$ is isomorphic to $O \times Z_{2}, D_{2 n}$, or $D_{n} \times Z_{2}$ according as $K$ is $K(3), K(n)(n \geq 4)$, or $C(n)$. Here $O$ denotes the octahedral group of order 24 , and $D_{n}$ denotes the dihedral group of order $2 n$.

Proof. (1) follows from Proposition I.1.4 together with the facts that (i) the cusp cross sections determined by the preceding horospherical cross sections of $S^{3}-K$ bound the same hyperbolic volumes and that (ii) the (Euclidean) center of $P(n)$ has the same hyperbolic distances from the horospherical cross sections. 
(2) By (1), $\operatorname{Isom}\left(S^{3}-K\right)$ is isomorphic to the combinatorial automorphism group of the decomposition, and it is easy to see that this group is isomorphic to the group in the proposition.

REMARK I.2.6. (1) The isometry group of the Borromean rings complement had been calculated by Riley [R3, Section 3]. One can also find the calculation of the symmetry group of the Borromean rings in [BS, Theorem 17.14] and [BZ, Example $2.2]$ by different methods.

(2) $\operatorname{Isom}\left(S^{3}-C(n)\right)$ had been calculated by Neumann-Reid [NR, Theorem 5.1 (iv)] by a different method.

Suppose $K=C^{\prime}(n)$. Then $S^{3}-K$ shares the same fundamental regions with $S^{3}-C(n)$ (cf. [Wi]), but the condition (i) in the above proof does not hold. So the above decomposition of $S^{3}-K$ is not canonical, however we can also see that it is a Euclidean decomposition. This implies that the subgroup of $\operatorname{Isom}\left(S^{3}-K\right)$ preserving the cusp around the "central" component (i.e., the component whose linking number with any other component is $n$ ) is isomorphic to $D_{2 n}$. From this fact, we can see that the symmetry group of $K$ is isomorphic to $D_{2 n}$. By using SnapPea, we can see that $\operatorname{Isom}\left(S^{3}-K\right) \cong D_{2 n}$ and that the canonical decomposition of $S^{3}-K$ is a subdivision of the preceding decomposition. In fact, it is obtained by subdividing each $P(n)$ as illustrated in Figure I.2.7 (a), and its edges can be seen as illustrated in Figure 1.2.7 (b).

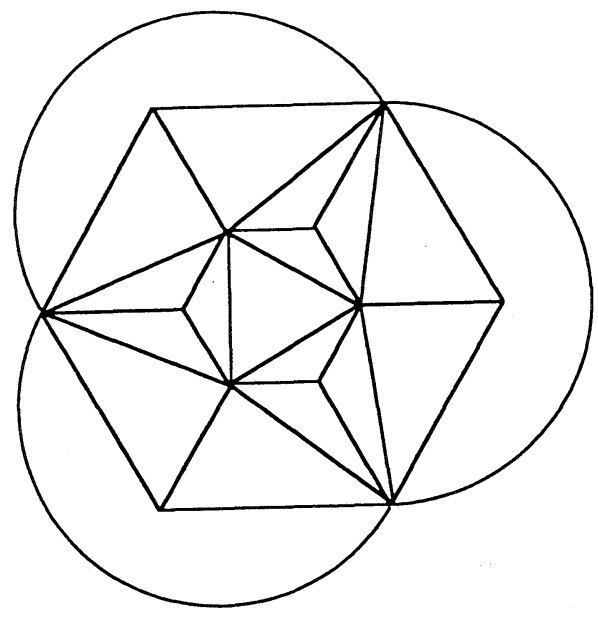

(a)

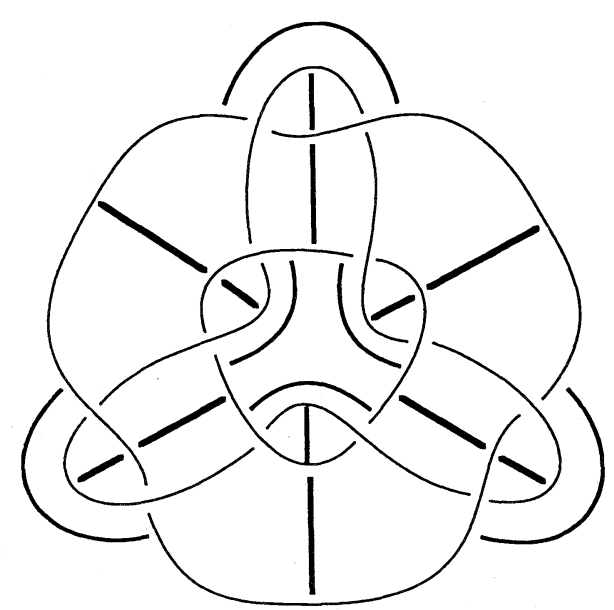

(b)

Figure I.2.7 
To close this section, we note the following proposition, which explains why the canonical decompositions given in this section are not ideal triangulations:

Proposition I.2.7. Suppose a cusped hyperbolic 3-manifold $M$ admits an isometry $f$ such that the period of $f$ is greater than 2 and that $\operatorname{Fix}(f)$ contains a circle component. Then the canonical decomposition $\mathcal{D}$ of $M$ is not an ideal triangulation.

Proof. Let $F$ be a circle component of $\operatorname{Fix}(f)$. Since the 1-skeleton of $\mathcal{D}$ is a disjoint union of infinite geodesics, $F$ is not contained in it. Suppose $F$ is contained in the 2-skeleton of $\mathcal{D}$. Then there is a 2-cell, say $\tau$, of $\mathcal{D}$, such that $F \cap \operatorname{int}(\tau)$ contains a 1-dimensional component. Since $f$ preserves $\mathcal{D}$, we have $f(\tau)=\tau$. So, the restriction of $f^{2}$ to $\tau$ is the identity. Since $f^{2}$ is orientation-preserving, we have $f^{2}=1$, a contradiction. Hence, $F$ is not contained in the 2 -skeleton of $\mathcal{D}$. This implies that $F$ intersects the interior of some 3-cell, say $\sigma$, of $\mathcal{D}$. Since $f$ preserves $\mathcal{D}$, we see $f(\sigma)=\sigma$. By the assumption, the restriction of $f$ to $\sigma$ has period greater than 2. Suppose $\sigma$ is an ideal simplex. Then, by the above fact and the fact that $f$ is orientation-preserving, we see that $f$ acts on the set of ideal vertices of $\sigma$ as a cyclic permutation of order 3 . (Note that an isometry of an ideal simplex is determined by its action on the set of vertices.) This implies that $\sigma$ is a regular ideal simplex, and $\operatorname{int}(\sigma) \cap \operatorname{Fix}(f)$ is a half line passing through an ideal vertex of $\sigma$. This contradicts the fact that $F$ is a circle. Hence $\sigma$ is not an ideal simplex, so $\mathcal{D}$ is not an ideal triangulation.

\section{Chapter II. Presumably canonical decompositions of 2-bridge link complements}

\section{II.1. Conway sphere and the diagram of $S L(2, \mathbb{Z})$}

Let $\Gamma$ be the group of isometries of $\mathbb{R}^{2}$ generated by $\pi$-rotations about the points of the integral lattice $\mathbb{Z}^{2}$. The pair $\left(\mathbb{R}^{2}, \mathbb{Z}^{2}\right) / \Gamma$ is denoted by $\left(S^{2}, P\right)$ and is called the Conway sphere. Then the following is well-known (cf. [HT], [BS, Chapter 9]).

(i) The isotopy classes of circles in $S^{2}-P$ separating $P$ into pairs are in one-to-one correspondence with $\mathbb{Q} \cup\{1 / 0\}$.

(ii) The isotopy classes of arcs in $S^{2}-P$ joining one given puncture to any of the other three punctures are in one-to-one correspondence with $\mathbb{Q} \cup\{1 / 0\}$.

In either case, a representative of the isotopy class corresponding to $b / a \in$ $\mathbb{Q} \cup\{1 / 0\}$ is the projection of a line in $\mathbb{R}^{2}$ of slope $b / a$ (the line being disjoint from $\mathbb{Z}^{2}$ in (i) and intersecting $\mathbb{Z}^{2}$ in (ii)). The number $b / a \in \mathbb{Q} \cup\{1 / 0\}$ associated to a circle or an arc in (i) and (ii) is called its slope.

For each matrix $A=\left(\begin{array}{ll}a & b \\ c & d\end{array}\right)$ in $G L(2, \mathbb{Z})$, consider the linear automorphism 
of $\left(\mathbb{R}^{2}, \mathbb{Z}^{2}\right)$ defined by $A(x, y)=(a x+b y, c x+d y)$. Since $A$ is $\Gamma$-equivariant, it induces an automorphism of $\left(S^{2}, P\right)$, which we denote by the same symbol $A$. Let $\widehat{A}$ be the transformation on $\mathbb{Q} \cup\{1 / 0\}$ defined by $\widehat{A}(s)=\frac{c+d s}{a+b s}$. Then $A$ sends arcs and loops in $\left(S^{2}, P\right)$ of slope $s$ to those of slope of $\widehat{A}(s)$. Note that $\widehat{A}$ extends to an automorphism of the hyperbolic plane $\mathbb{H}^{2}=\{z \in \mathbb{C} \mid \operatorname{Im}(z)>0\}$ and the circle at infinity $\partial \mathbb{H}^{2}=\mathbb{R} \cup\{1 / 0\}$ by the following rule:

$$
\widehat{A}(z)= \begin{cases}\frac{c+d z}{a+b z} & \text { if } \operatorname{det} A=1, \\ \frac{c+d \bar{z}}{a+b \bar{z}} & \text { if } \operatorname{det} A=-1\end{cases}
$$

Consider the ideal triangle in $\mathbb{H}^{2}$ spanned by the vertices $\{0 / 1,1 / 0,1 / 1\}$. Then the translates of this ideal triangle by the action of $S L(2, \mathbb{Z})$ forms a tessellation of $\mathbb{H}^{2}$. This is called the diagram of $P S L(2, \mathbb{Z})$. Each ideal triangle $T$ in the diagram of $P S L(2, \mathbb{Z})$ determines a $\Gamma$-equivariant triangulation $\widetilde{\Delta}(T)$ of $\left(\mathbb{R}^{2}, \mathbb{Z}^{2}\right)$ and a triangulation $\Delta(T)$ of $\left(S^{2}, P\right)$ as follows. By definition, the vertex set of $T$ is of the form $\{c / a, d / b,(c+d) /(a+b)\}$, where $a, b, c$, and $d$ are integers such that g.c.d. $\{a, c\}=$ g.c.d. $\{b, d\}=$ g.c.d. $\{a+b, c+d\}=1$. Then $\widetilde{\Delta}(T)$ is the triangulation of $\left(\mathbb{R}^{2}, \mathbb{Z}^{2}\right)$ spanned by the three integral vectors $(a, c),(b, d)$, and $(a+b, c+d)$, and $\Delta(T)$ is the quotient of $\widetilde{\Delta}(T)$ (see Figure II.1.1).

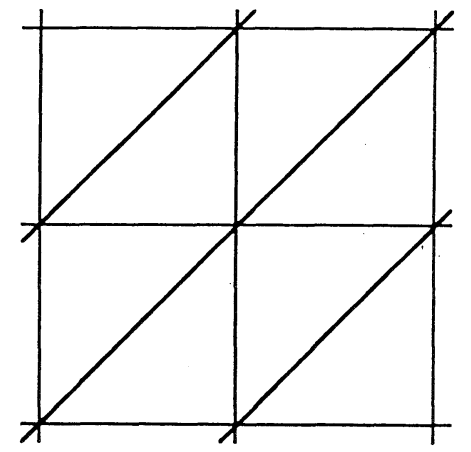

$\widetilde{\Delta}(T)$ with $T=<1 / 0,0 / 1,1 / 1>$

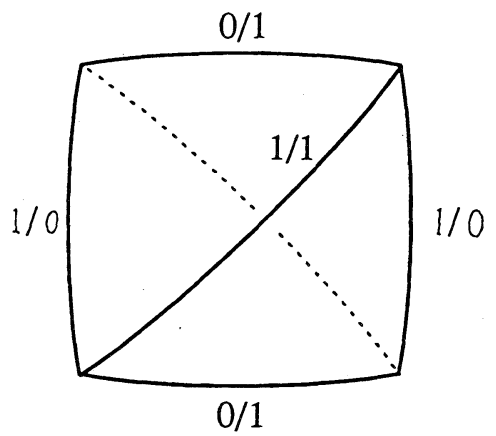

$\Delta(T)$

Figure II.1.1

It should be noted that $A(\widetilde{\Delta}(T))=\widetilde{\Delta}(\widehat{A}(T))$ and $A(\Delta(T))=\Delta(\widehat{A}(T))$ for any matrix $A$ in $G L(2, \mathbb{Z})$ and for any ideal triangle $T$ in the diagram of $S L(2, \mathbb{Z})$.

\section{II.2. Construction of the ideal triangulation}

First, we recall the definitions of a trivial tangle and a rational tangle. A trivial tangle is a pair $\left(B^{3}, t\right)$, where $B^{3}$ is a 3 -ball and $t$ is a union of two $\operatorname{arcs}$ in $B^{3}$ which 
is parallel to a union of two mutually disjoint $\operatorname{arcs}$ in $\partial B^{3}$. A meridian $m$ of $\left(B^{3}, t\right)$ is a simple loop on $\partial B^{3}-t$ which bounds a disk in $B^{3}$ separating the components of $t$ (see Figure II.2.1). A rational tangle is a trivial tangle $\left(B^{3}, t\right)$ endowed with a homeomorphism from $\partial\left(B^{3}, t\right)$ to the Conway sphere $\left(S^{2}, P\right)$. The slope of a rational tangle is defined to be the slope of its meridian.

Let $K(p, q)$ be the 2-bridge link of type $(p, q)$. Then it is obtained as the "sum" of the rational tangles of slopes $1 / 0$ and $q / p$; i.e., $\left(S^{3}, K(p, q)\right)$ is obtained from the rational tangles of slopes $1 / 0$ and $q / p$ by identifying their boundaries through the identity map. (Note that each of the boundaries of the rational tangles are identified with the Conway sphere, so the term "identity map" has a well-defined meaning.)

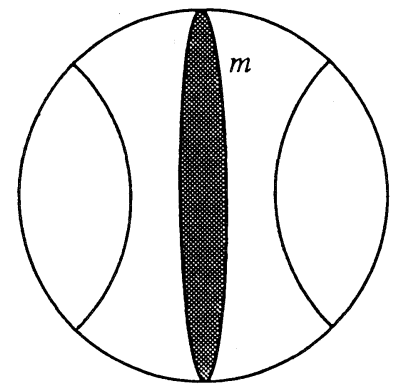

$\left(B^{3}, t\right)$

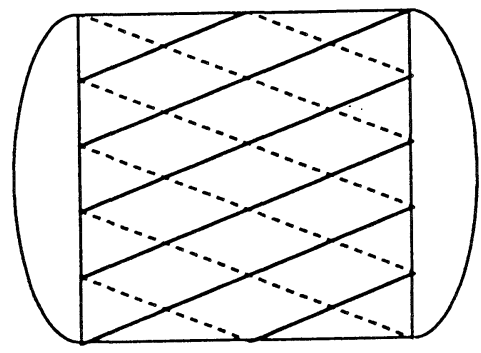

$S(5,2)$

Figure II.2.1

In the following, we consider only hyperbolic 2-bridge links; i.e., we exclude $(2, n)$-torus links from our consideration. So we assume $q \not \equiv \pm 1(\bmod p)$. By reversing the orientations if necessary, we may assume further $1<q<p / 2$. Then $q / p$ has the continued fraction expansion

$$
\frac{q}{p}=\left[a_{1}, a_{2}, \ldots, a_{n}\right]=\frac{1}{a_{1}+\frac{1}{a_{2}+\cdots+\frac{1}{a_{n}}}},
$$

such that $n \geq 2, a_{i}>0(1 \leq i \leq n), a_{1} \geq 2$ and $a_{n} \geq 2$. Then we denote $K(p, q)$ also by $K\left[a_{1}, \ldots, a_{n}\right]$. Put $c_{k}=\sum_{i=1}^{k} a_{i}(0 \leq k \leq n)$ and $c=c_{n}$. Let $\Im=\left\{T_{1}, T_{2}, \ldots, T_{c}\right\}$ be the sequence of ideal triangles in the diagram of $S L(2, \mathbb{Z})$ in $\mathbb{H}^{2}$ as illustrated in Figure II.2.2.

Let $\left\{r=r_{1}, r_{2}, r_{3}\right\}$ [resp. $\left.\left\{r^{\prime}=r_{1}^{\prime}, r_{2}^{\prime}, r_{3}^{\prime}\right\}\right]$ be the vertices of the ideal simplex $T_{2}$ [resp. $T_{c-1}$ ] satisfying the following conditions (see Figure II.2.3).

(i) The edge $\overline{r_{2} r_{3}}$ [resp. $\left.\overline{r_{2}^{\prime} r_{3}^{\prime}}\right]$ is the common edge of $T_{2}$ and $T_{1}$ [resp. $T_{c-1}$ and $T_{c}$. 
(ii) The edge $\overline{r_{1} r_{3}}$ [resp. $\left.\overline{r_{1}^{\prime} r_{3}^{\prime}}\right]$ is the common edge of $T_{2}$ and $T_{3}$ [resp. $T_{c-1}$ and $\left.T_{c-2}\right]$.

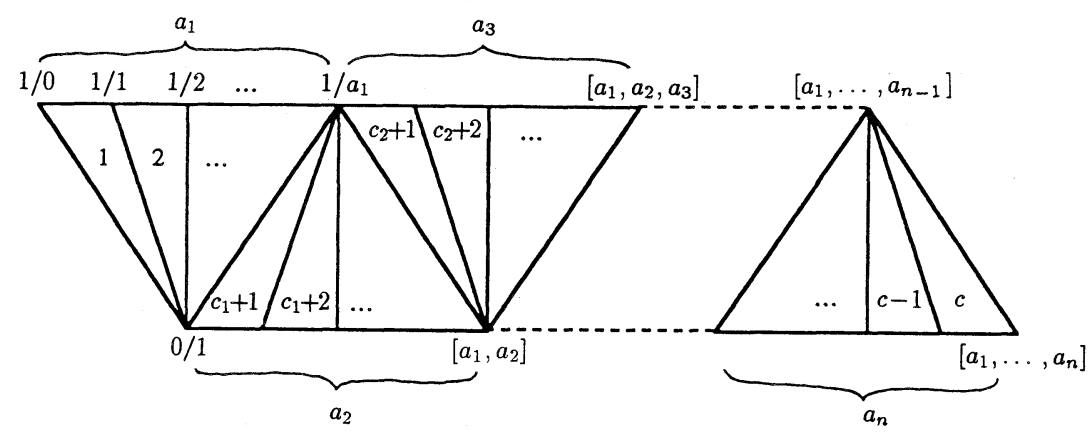

Figure II.2.2

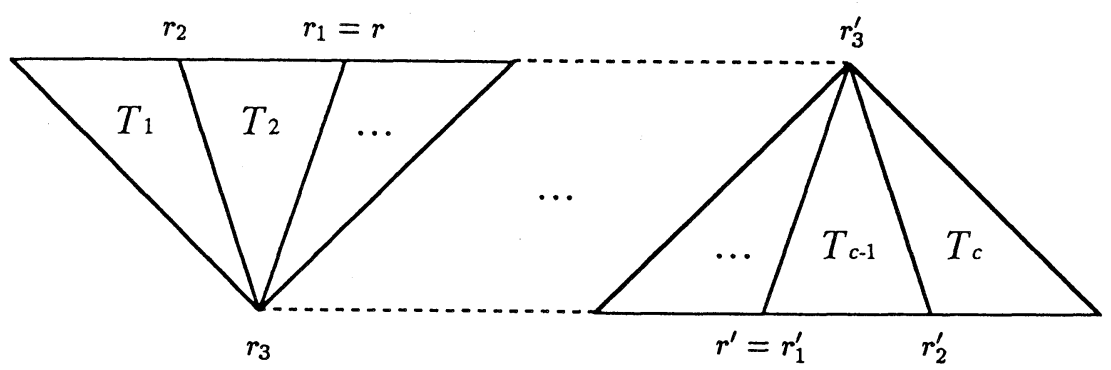

Figure II.2.3

Note that the triangles $\left\{T_{2}, \ldots, T_{c-1}\right\}$ have a common vertex $r_{c m}$, if and only if one of the following conditions holds. (See Figure II.2.4 (a), (b) and (c)). $r_{1}=r_{1}^{\prime}$

(II.2.1) $n=3$ and $a_{1}=a_{3}=2$. Then there is a unique common vertex

(II.2.2) $n=2$ and precisely one of $a_{1}$ and $a_{2}$ is equal to 2 . Then there is a unique common vertex, and it is equal to $r_{1}=r_{3}^{\prime}$ or $r_{3}=r_{1}^{\prime}$ according as $a_{1}=2$ or $a_{2}=2$.

(II.2.3) $n=2$ and $a_{1}=a_{2}=2$. Then there are two common vertices $r_{1}=r_{3}^{\prime}$ and $r_{3}=r_{1}^{\prime}$.

If one of the above conditions holds, we say that $q / p$ is exceptional, and the rational numbers associated to the common vertices are referred as the common slopes. [There is a common vertex in case $n=1$ too, however this does not occur by our assumption that $q \not \equiv \pm 1(\bmod p)$.] 


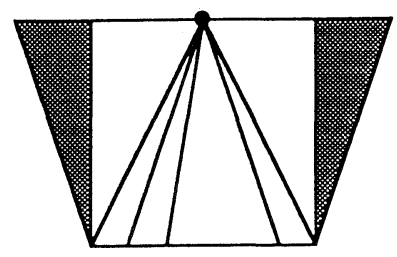

(a)

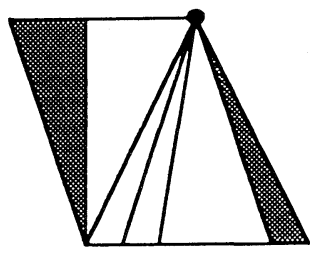

(b)

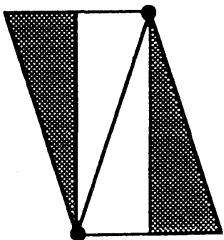

(c)

Figure II.2.4
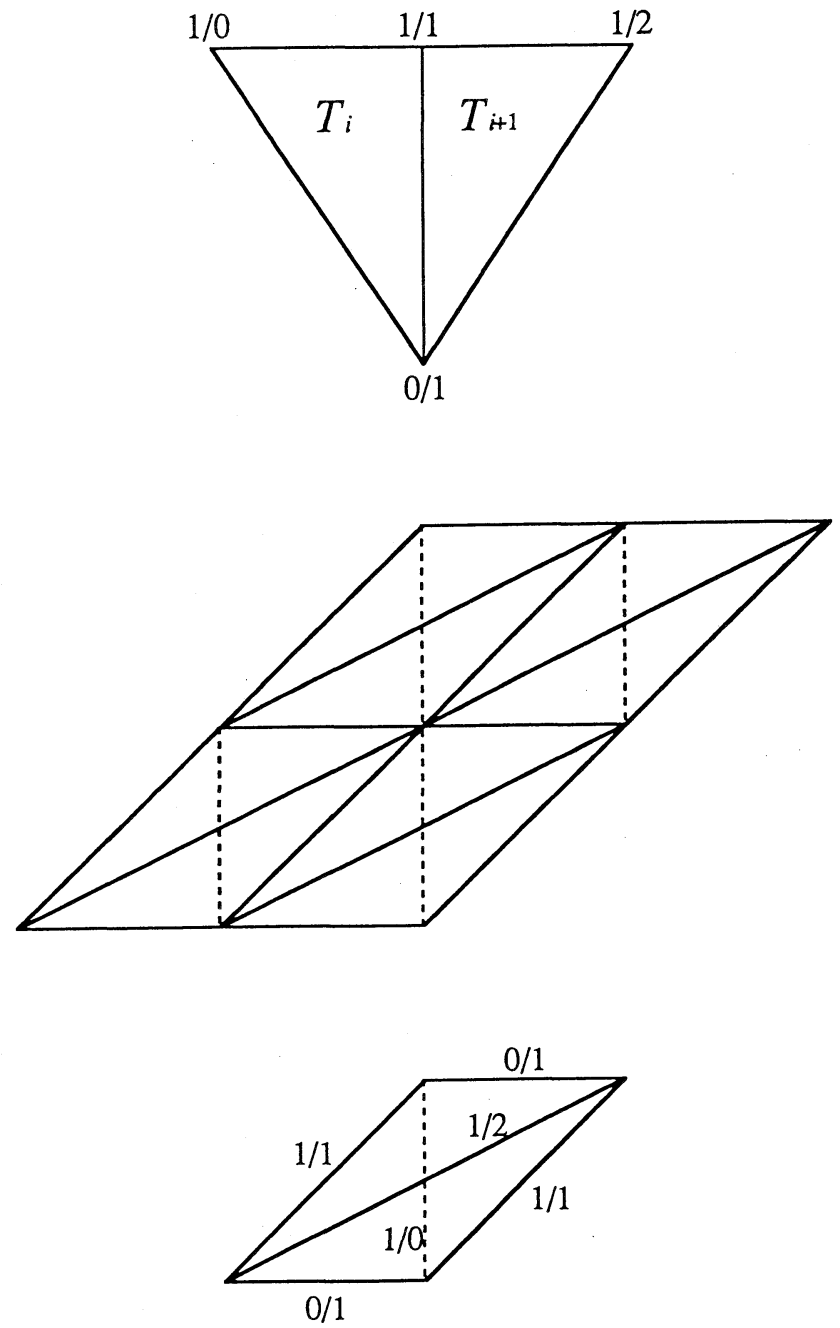

Figure II.2.5 
For each $i(2 \leq i \leq c-1)$, consider the $\Gamma$-equivariant triangulation $\widetilde{\Delta}_{i}=\widetilde{\Delta}\left(T_{i}\right)$ of $\left(\mathbb{R}^{2}, \mathbb{Z}^{2}\right)$. Since $T_{i}$ and $T_{i+1}$ have two vertices in common, $\widetilde{\Delta}_{i}$ and $\widetilde{\Delta}_{i+1}$ have two edge slopes in common. If we superimpose $\widetilde{\Delta}_{i+1}$ upon $\widetilde{\Delta}_{i}$, we see an array of tetrahedra, whose bottom faces form $\widetilde{\Delta}_{i}$ and whose top faces form $\widetilde{\Delta}_{i+1}$ (see Figure II.2.5). We denote this array of tetrahedra by $\widetilde{\mathcal{D}}_{i}$.

Consider $\widetilde{\mathcal{D}}_{i}$ for $i=2,3, \ldots, c-2$, and stack them up in order. Then we obtain a set of layers of tetrahedra, whose bottom faces form $\widetilde{\Delta}_{2}$ and whose top faces form $\widetilde{\Delta}_{c-1}$. By deleting the vertices $\mathbb{Z}^{2}$ from the above set of layers of tetrahedra, we obtain a decomposition $\widetilde{\mathcal{D}}$ of $\left(\mathbb{R}^{2}-\mathbb{Z}^{2}\right) \dot{\times} I$ into ideal tetrahedra. Here, $\left(\mathbb{R}^{2}, \mathbb{Z}^{2}\right) \dot{\times} I$ is the pair of topological spaces defined as follows: If $q / p$ is not exceptional, then $\left(\mathbb{R}^{2}, \mathbb{Z}^{2}\right) \dot{\times} I=\left(\mathbb{R}^{2}, \mathbb{Z}^{2}\right) \times I$. Otherwise, $\left(\mathbb{R}^{2}, \mathbb{Z}^{2}\right) \dot{\times} I=\left\{\left(\mathbb{R}^{2}, \mathbb{Z}^{2}\right) \times I\right\} / \dot{\sim}$, where $\dot{\sim}$ collapses $x \times I$ to a point for each point $x$ on the lines in $\mathbb{R}^{2}$ of the common slopes which intersect $\mathbb{Z}^{2}$.

Let $\Gamma$ also denote the group of homeomorphisms of $\left(\mathbb{R}^{2}, \mathbb{Z}^{2}\right) \dot{\times} I$ generated by $\pi$-rotations about $p \dot{\times} I$, where $p$ runs over $\mathbb{Z}^{2}$. Put $\left(S^{2}, P\right) \dot{\times} I=\left\{\left(\mathbb{R}^{2}, \mathbb{Z}^{2}\right) \dot{\times} I\right\} / \Gamma$. Then $\Gamma$ preserves the decomposition $\widetilde{\mathcal{D}}$, and therefore its quotient $\widehat{\mathcal{D}}$ determines an ideal triangulation of $\left(S^{2}-P\right) \dot{\times} I$. The ideal triangulations of the faces $\left(S^{2}-P\right) \dot{\times} 0$ and $\left(S^{2}-P\right) \dot{\times} 1$ induced by $\widehat{\mathcal{D}}$ are given by the quotients $\Delta_{2}=\widetilde{\Delta}_{2} / \Gamma$ and $\Delta_{c-1}=$ $\widetilde{\Delta}_{c-1} / \Gamma$ respectively.

Next, we define certain equivalence relations on $\Delta_{2}$ and on $\Delta_{c-1}$. To do this, remember the vertex $r=r_{1}$ of $T_{2}$ [resp. the vertex $r^{\prime}=r_{1}^{\prime}$ of $T_{c-1}$ ], and let $\sim_{r}$ [resp. $\sim_{r^{\prime}}$ ] be the equivalence relation on $\widetilde{\Delta}_{2}$ [resp. $\widetilde{\Delta}_{c-1}$ ] generated by the following elementary relation: Let $\widetilde{\tau}$ and $\widetilde{\tau}^{\prime}$ be simplices of $\widetilde{\Delta}_{2}\left[\right.$ resp. $\left.\widetilde{\Delta}_{c-1}\right]$ such that $\widetilde{\tau} \cap \widetilde{\tau}^{\prime}$ is an edge of slope $r$ [resp. $r^{\prime}$ ], and let $f$ be a linear homeomorphism from $\widetilde{\tau}$ to $\widetilde{\tau}^{\prime}$ which is the identity on the common edge; then $x \sim_{r} f(x)$ [resp. $x \sim_{r^{\prime}} f(x)$ ]. Since these relations are $\Gamma$-equivariant, they induce equivalence relations on $\Delta_{2}$ and $\Delta_{c-1}$, which we denote by the same symbols $\sim_{r}$ and $\sim_{r^{\prime}}$. Let $\mathcal{D}$ be the ideal simplicial complex obtained from $\widehat{\mathcal{D}}$ by performing the identifications induced by the relations $\sim_{r}$ and $\sim_{r^{\prime}}$ on its faces $\Delta_{2}$ and $\Delta_{c-1}$ respectively. If necessary, we denote $\mathcal{D}$ by $\mathcal{D}(p, q)$ or $\mathcal{D}\left[a_{1}, \ldots, a_{n}\right]$. We have the following theorem:

TheOREM II.2.4. $\mathcal{D}=\mathcal{D}(p, q)$ gives an ideal triangulation of $S^{3}-K(p, q)$.

To prove this theorem, we need the following lemma:

LEMMA II.2.5. Let $T$ be an ideal simplex in the diagram of $S L(2, \mathbb{Z})$ with vertices $\left\{s_{1}, s_{2}, s_{3}\right\}$. Let $T^{*}$ be the ideal simplex in the diagram of $S L(2, \mathbb{Z})$ which has the vertices $\left\{s_{2}, s_{3}\right\}$ in common with $T$, and let $s_{1}^{*}$ be the remaining vertex of $T^{*}$. Consider the space $\left(S^{2}, P\right) \times I=\left\{\left(\mathbb{R}^{2}, \mathbb{Z}^{2}\right) \times I\right\} / \Gamma$ and the triangulation $\Delta(T)$ of its face $\left(S^{2}, P\right) \times 0$. Let $\sim_{s_{1}}$ be the equivalence relation on $\left(S^{2}, P\right) \times 0$ determined by the slope $s_{1}$ as in the above. Put $(B, k)=\left\{\left(S^{2}, P\right) \times I\right\} / \sim_{s_{1}}$. Then $(B, k)$ is a rational tangle of slope $s_{1}^{*}$; i.e., $(B, k)$ is a trivial tangle, and the image of a line on 
$\mathbb{R}^{2} \times 1$ of slope $s_{1}^{*}$ disjoint from $\mathbb{Z}^{2}$ forms a meridian of $(B, k)$.

Proof. We may suppose $s_{1}=1 / 0, s_{2}=0 / 1, s_{3}=1 / 1$, and $s_{1}^{*}=1 / 2$. Figure II.2.6 describes the equivalence relation $\sim_{r_{1}}$, and Figure II.2.7 shows that $(B, k)$ is a trivial tangle. Its meridian is depicted in Figure II.2.8, and we see that its slope is $1 / 2=s^{*}$.
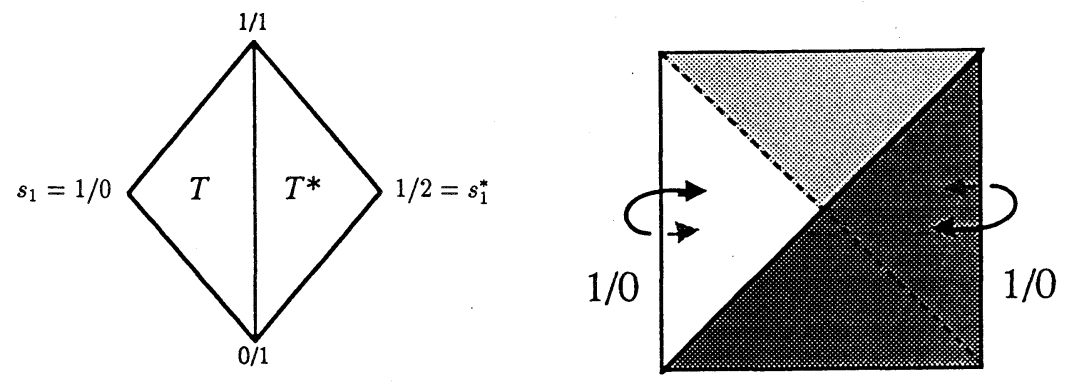

Figure II.2.6
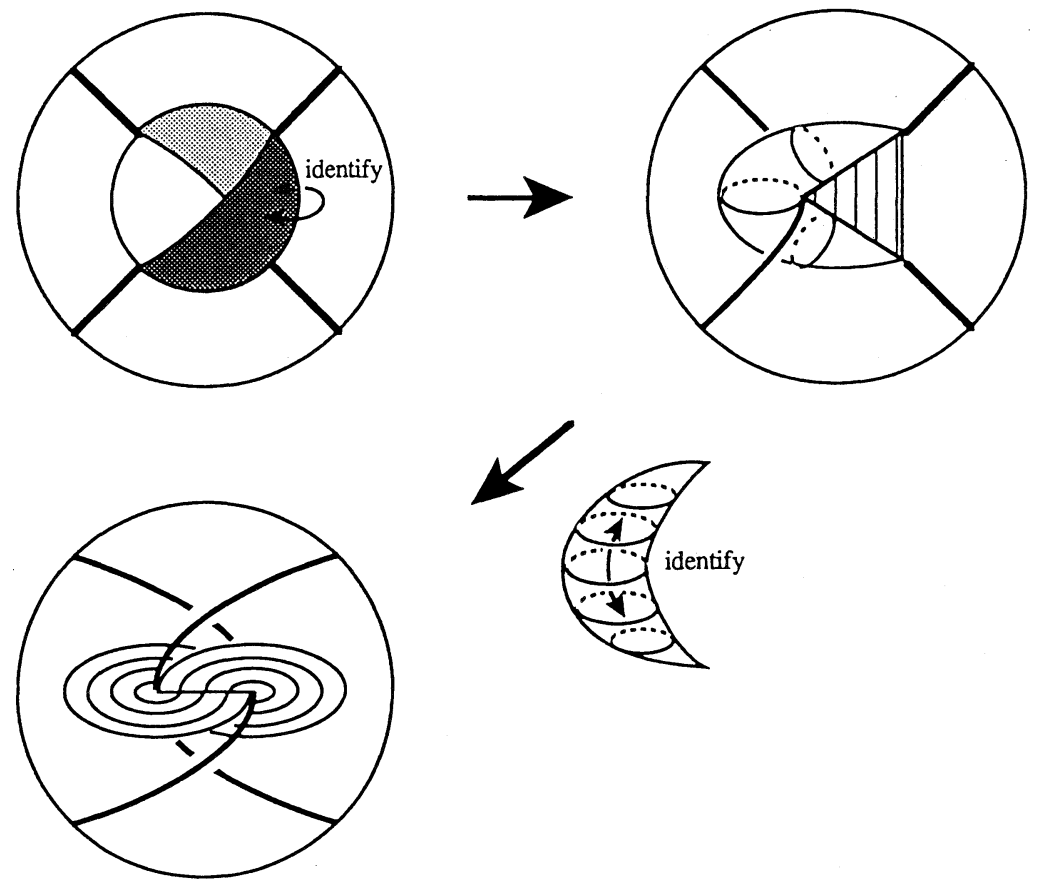

Figure II.2.7 

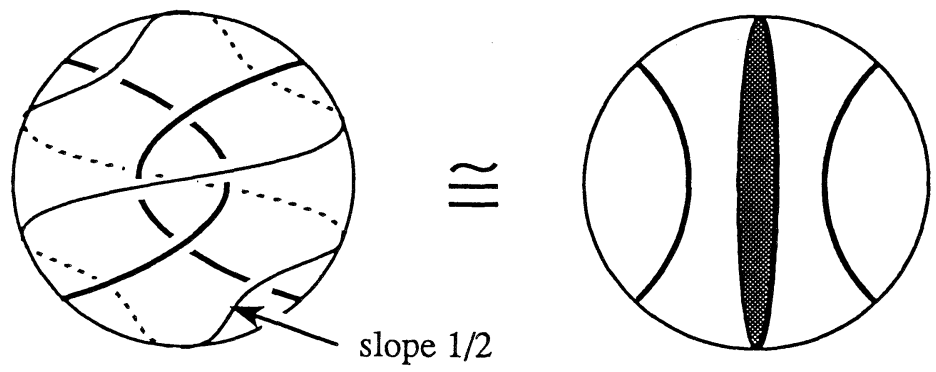

Figure II.2.8

Remark II.2.6. For $i=1,2,3$, let $\gamma_{i}$ be the union of two $\operatorname{arcs}$ on $\left(S^{2}, P\right)$ which is the image of the union of the lines of slope $s_{i}$ in $\mathbb{R}^{2}$ that intersect $\mathbb{Z}^{2}$. Let $\Psi:\left(S^{2}, P\right) \times I \rightarrow(B, k)$ be the projection. Then we have the following:

(1) $\Psi\left(\gamma_{1} \times I\right)$ is a union of two once-pinched disks, such that $\Psi\left(\gamma_{1} \times I\right) \cap k=$ $\Psi\left(\partial \gamma_{1} \times I\right)$ and $\Psi\left(\gamma_{1} \times I\right) \cap \partial B=\Psi\left(\gamma_{1} \times 1\right)$ (see Figure II.2.9 (a)).

(2) Suppose $i=2$ or 3 . Then $\Psi\left(\gamma_{i} \times I\right)$ is a disk, such that $\Psi\left(\gamma_{i} \times I\right) \cap k=$ $\Psi\left(\partial \gamma_{i} \times I\right)$ and $\Psi\left(\gamma_{i} \times I\right) \cap \partial B=\Psi\left(\gamma_{i} \times 1\right)$ (see Figure II.2.9 (b)).

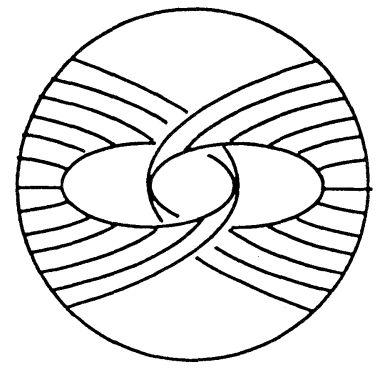

(a)

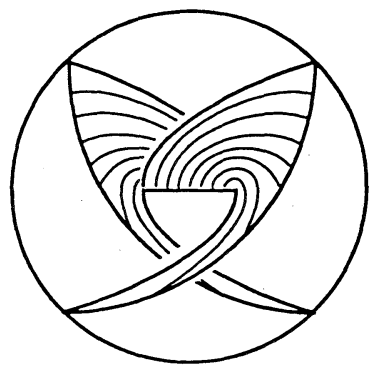

(b)

Figure II.2.9

We now prove Theorem II.2.4. Let $(M, K)$ be the space obtained from $\left(S^{2}, P\right) \dot{\times} I$ by performing the identifications on its faces $\left(S^{2}, P\right) \times 0$ and $\left(S^{2}, P\right) \times 1$ induced by the relations $\sim_{r}$ and $\sim_{r^{\prime}}$ respectively. Then $\mathcal{D}$ gives an ideal triangulation of $M-K$. Thus we have only to show $M-K \cong S^{3}-K(p, q)$.

CASE 1. $q / p$ is not exceptional. For $i=0,1$, let $B_{i}$ be the closed up component of $M-\left(S^{2} \times\{1 / 2\}\right)$ containing the image of $S^{2} \times\{i\}$, and put $k_{i}=B_{i} \cap K$. Then by Lemma II.2.5, $\left(B_{0}, k_{0}\right)$ and $\left(B_{1}, k_{1}\right)$, respectively, are the rational tangles of slopes $1 / 0$ and $q / p$. Hence $(M, K)$ is homeomorphic to $\left(S^{3}, K(p, q)\right)$, and we 
obtain the desired result.

CASE 2. $q / p$ is exceptional. Let $(\widetilde{M}, \widetilde{K})$ be the space obtained from $\left(S^{2}, P\right) \times$ $I$ (instead of $\left.\left(S^{2}, P\right) \dot{\times} I\right)$ by performing the identification induced by $\sim_{r}$ and $\sim_{r^{\prime}}$, and let $\Phi:\left(S^{2}, P\right) \times I \rightarrow(\widetilde{M}, \widetilde{K})$ be the projection. Then the previous argument shows $(\widetilde{M}, \widetilde{K}) \cong\left(S^{3}, K(p, q)\right)$. Let $\gamma$ be the image, in $\left(S^{2}, P\right)$, of the union of the lines in $\mathbb{R}^{2}$ of the common slopes that intersect $\mathbb{Z}^{2}$. By the construction, we see $(M, K) \cong(\widetilde{M}, \widetilde{K}) / \dot{\sim}$, where $\dot{\sim}$ collapses $\Psi(x \times I)$ to a point for each $x \in \gamma$. We have only to show that $\dot{\sim}$ does not change the topological type of $\widetilde{M}-\widetilde{K}$. But, this follows from the following facts, which can be proved by using Remark II.2.6.

(1) Suppose (II.2.1) is satisfied. Then $\Phi(\gamma \times I)$ is a disjoint union of two twice pinched disks, such that $\Phi(\gamma \times I) \cap K=\Phi(\partial \gamma \times I)$.

(2) Suppose (II.2.2) is satisfied. Then $\Phi(\gamma \times I)$ is a pinched disk, such that $\Phi(\gamma \times I) \cap K=\Phi(\partial \gamma \times I)$.

(3) Suppose (II.2.3) is satisfied. Then $\Phi(\gamma \times I)$ is a union of two twice pinched disks with mutually disjoint interiors, such that $\Phi(\gamma \times I) \cap K=\Phi(\partial \gamma \times I)$.

REMARK II.2.7. If $n=1$ (i.e., $q=1$ ), then $\Phi(\gamma \times I)$ is an annulus or a Möbius band. So, $(\widetilde{M}-\widetilde{K}) / \dot{\sim}$ is not homeomorphic to $\widetilde{M}-\widetilde{K}$.

\section{II.3. Isomorphism problem and the automorphism group of $\mathcal{D}$}

In this section, we solve the isomorphism problem of the combinatorial complexes $\mathcal{D}(p, q)$ 's and determine the automorphism group of $\mathcal{D}(p, q)$. The solutions for the case where $p$ is odd are easily obtained if we use the following facts: (i) The 2-bridge knots and links are classified by Schubert [Sc], (ii) the symmetry groups of the 2-bridge knots and links are determined by Bonahon and Siebenmann [BS] (cf. $[\mathrm{Sa} 1,2]$ ), and (iii) (2-bridge) knots are determined by their complements (see [Ta1, GL]). On the other hand, in this section, we solve these problems without appealing to these facts. This is because the authors do not know whether a 2-bridge link is determined by its complement among the 2-bridge links; furthermore, if it were proved that the triangulation $\mathcal{D}$ is actually the canonical one, then our solution gives an affirmative answer to this question and an alternative proof to the above facts. By the way, we give intuitive pictures of the decomposition $\mathcal{D}$ (Figure II.3.3).

First, we prove the following:

TheOREM II.3.1. The sequence of positive integers, $a_{1}, a_{2}, \ldots, a_{n}$, up to inversion, is canonically determined by $\mathcal{D}\left[a_{1}, a_{2}, \ldots, a_{n}\right]$. That is, two complexes $\mathcal{D}\left[a_{1}, \ldots, a_{n}\right]$ and $\mathcal{D}\left[a_{1}^{\prime}, \ldots, a_{n^{\prime}}^{\prime}\right]$ are are combinatorially equivalent, if and only if $\left(a_{1}, \ldots, a_{n}\right)$ is equal to $\left(a_{1}^{\prime}, \ldots, a_{n^{\prime}}^{\prime}\right)$ up to inversion. Hence, $\mathcal{D}(p, q)$ and $\mathcal{D}\left(p^{\prime}, q^{\prime}\right)$ are combinatorially equivalent, if and only if $p=p^{\prime}$ and either $q \equiv q^{\prime}(\bmod p)$ or $q q^{\prime} \equiv \pm 1(\bmod p)$ 
Proof. We number the vertices of $\Im-\left\{T_{1}, T_{c}\right\}$ as illustrated in Figure II.3.1.

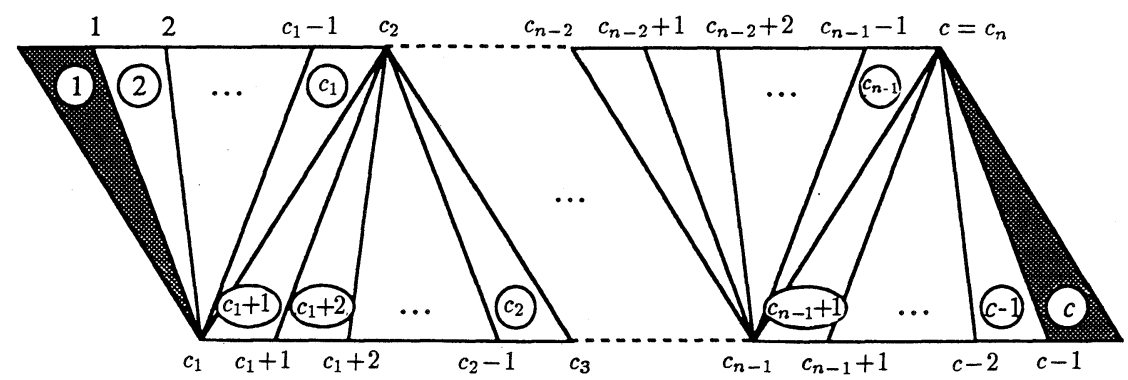

Figure II.3.1

We denote the $i$-th vertex by $s(i)$. Then each $s(i)(1 \leq i \leq c)$ is characterized by the following properties:

(1) If $1 \leq i \leq c-2$, then $s(i)$ is the vertex of $T_{i+1}$ which is not a vertex of any $T_{j}(j \geq i+2)$.

(2) $s(c-1)$ and $s(c)$ are vertices of $T_{c-1}$, and $s(c-1)$ is incident on only two triangles of $\Im$, whereas $s(c)$ is incident on $a_{n}+1$ triangles of $\Im$.

Let $\varepsilon(i)(1 \leq i \leq c)$ be the union of the edges of $\mathcal{D}$ which are the images of the edges of $\widetilde{\mathcal{D}}$ of slope $s(i)$. Let $\tau(i)(2 \leq i \leq c-1)$ be the union of the faces of $\mathcal{D}$ which are the images of the faces of $\widetilde{\mathcal{D}}$ determined by $\widetilde{\Delta}_{i}$. Let $\sigma(i)(2 \leq i \leq c-2)$ be the union of the tetrahedra of $\mathcal{D}$ which are the images of the tetrahedra of $\widetilde{\mathcal{D}}_{i}$. Then we see

(1) Each $\tau(i)(3 \leq i \leq c-2)$ consists of four faces, and each of $\tau(2)$ and $\tau(c-1)$ consists of two faces.

(2) Each $\sigma(i)(2 \leq i \leq c-2)$ consists of two tetrahedra. For each $i(2 \leq i \leq$ $c-3)$, each tetrahedron of $\sigma(i)$ intersects each tetrahedron of $\sigma(i+1)$ in a face belonging to $\tau(i+1)$. Each tetrahetron of $\sigma(2)$ [resp. $\sigma(c-2)]$ intersects the other tetrahedron of $\sigma(2)$ [resp. $\sigma(c-2)$ ] in the faces belonging to $\tau(2)$ [resp. $\tau(c-1)]$.

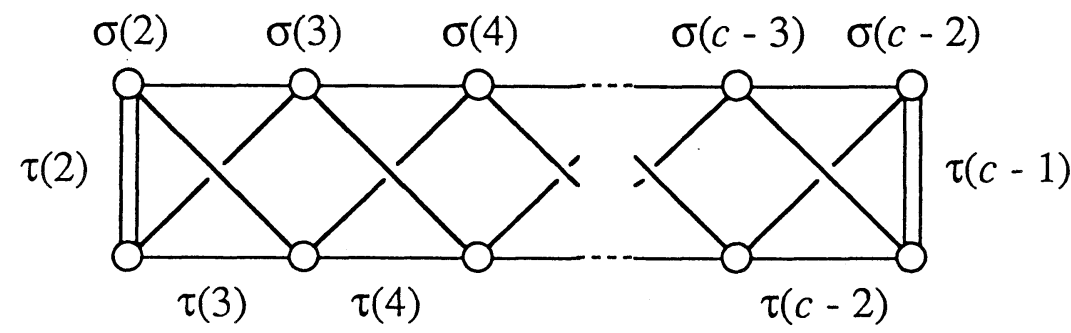

Figure II.3.2 
Let $\Omega$ be the 1 -skeleton of the dual complex of $\mathcal{D}$. Then, by the above facts, we see $\Omega$ is as illustrated in Figure II.3.2.

From the shape of $\Omega$, we see the following:

(1) The union $\tau(2) \cup \tau(c-1)$ is characteristic in $\mathcal{D}$; i.e., any combinatorial automorphism of $\mathcal{D}$ preserves $\tau(2) \cup \tau(c-1)$.

(2) The order $\tau(2), \tau(3), \ldots, \tau(c-1)$, and the order $\sigma(2), \sigma(3), \ldots, \sigma(c-2)$, up to inversion, are canonically determined by $\mathcal{D}$.

For the edge set, we can see the following:

(1) $\varepsilon(1)=\varepsilon\left(c_{1}\right)$ and $\varepsilon(c-1)=\varepsilon(c)$; each of them consists of one edge.

(2) For each $i \notin\left\{1, c_{1}, c-1, c\right\}, \varepsilon(i)$ consists of two edges.

(3) For each $i \notin\{1, c-1, c\}, \varepsilon(i)$ is characterized as the set of the edges which are incident on $\tau(i+1)$ and are not incident on $\tau(j)$ for any $j \geq i+2$. Note that $\tau(1)$ is characterized as $\tau\left(c_{1}\right)$, and $\tau(c-1)=\tau(c)$ is characterized as the set of the remaining edges.

(4) For each $i(1 \leq i \leq c)$, let $d(i)$ be the number of dihedral angles around an edge of $\varepsilon(i)$. Then it does not depend on the choice of an edge in $\varepsilon(i)$ and is given as follows.

$$
d(i)= \begin{cases}3 & \text { if } i=2 \neq c_{1} \\ 4 & \text { if } i \notin\{2, c-1\} \text { and } c_{k}+1 \leq i \leq c_{k+1}-1 \text { for some } k \\ 4 a_{1}+2 & \text { if } i=1 \text { or } c_{1} \\ 2 a_{k}+2 & \text { if } i=c_{k} \text { for some } k(2 \leq i \leq n-1) \\ 4 a_{n}+2 & \text { if } i=c-1 \text { or } c\end{cases}
$$

By (3), we see that the order $\varepsilon(2), \varepsilon(3), \ldots, \varepsilon(c-1)$, up to inversion, is canonically determined by $\mathcal{D}$. Thus, together with (4), we see the sequence $a_{1}, a_{2}, \ldots, a_{n}$, up to inversion, is canonically determined by $\mathcal{D}$. This completes the proof of the first half of Theorem II.3.1. The latter half follows from Lemma II.3.3.

In the link diagram, the edges and the faces of $\mathcal{D}$ can be seen as illustrated in Figure II.3.3.

Let $\operatorname{Aut}(\mathcal{D})$ be the group of the combinatorial automorphisms of the ideal simplicial complex $\mathcal{D}$, and let $\operatorname{Aut}^{+}(\mathcal{D})$ be the subgroup of $\operatorname{Aut}(\mathcal{D})$ consisting of the orientation-preserving automorphisms.

THEOREM II.3.2.

$$
\operatorname{Aut}^{+}(\mathcal{D}) \cong \begin{cases}\mathbb{Z}_{2} \oplus \mathbb{Z}_{2} & \text { if } q^{2} \not \equiv 1(\bmod p), \\ D_{4} & \text { if either } p \text { is odd and } q^{2} \equiv 1(\bmod p), \\ & \text { or } p \text { is even and } q^{2} \equiv p+1(\bmod 2 p), \\ \mathbb{Z}_{2} \oplus \mathbb{Z}_{2} \oplus \mathbb{Z}_{2} & \text { if } p \text { is even and } q^{2} \equiv 1(\bmod 2 p) .\end{cases}
$$




$$
\operatorname{Aut}(\mathcal{D}) \cong \begin{cases}\operatorname{Aut}^{+}(\mathcal{D}) & \text { if } q^{2} \not \equiv-1(\bmod p), \\ D_{4} & \text { if } q^{2} \equiv-1(\bmod p) .\end{cases}
$$

To prove this theorem, we need the following lemma. Though this is probably well-known, we give a proof at the end of this section.

\section{$K[4,3,2,3]$}

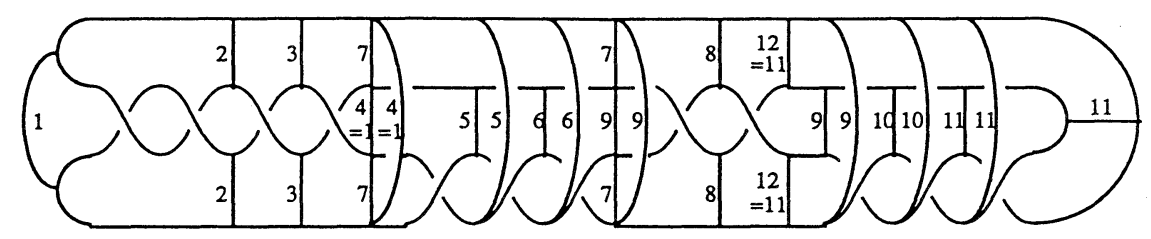

$\varepsilon(1)=\varepsilon(4)$ consists of one edge.

$\varepsilon(11)=\varepsilon(12)$ consists of one edge.

$\varepsilon(i)$ consists of two edges $(i \neq 1,4,11,12)$.

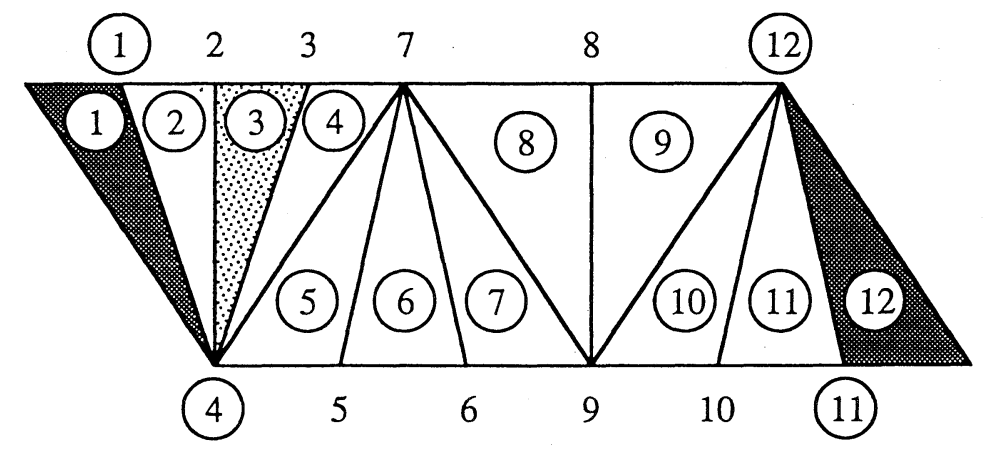

Two of the four faces in $\tau(3)$ :

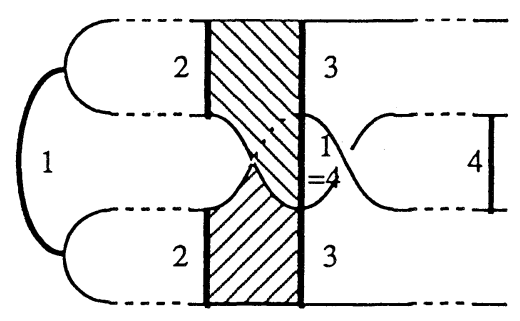

Figure II.3.3 
LEMMA II.3.3.

(1) The following are equivalent:

(a) $q^{2} \equiv-1(\bmod p)$.

(b) $n$ is even and the sequence $\left(a_{1}, \ldots, a_{n}\right)$ is symmetric (i.e., $a_{i}=$ $\left.a_{n+1-i}(1 \leq i \leq n)\right)$.

(2) The following are equivalent:

(a) Either (i) $p$ is odd and $q^{2} \equiv 1(\bmod p)$, or (ii) $p$ is even and $q^{2} \equiv p+1$ $(\bmod 2 p)$.

(b) $n$ is odd, $a_{(n+1) / 2}$ is odd, and $\left(a_{1}, \ldots, a_{n}\right)$ is symmetric.

(3) The following are equivalent:

(a) $p$ is even and $q^{2} \equiv 1(\bmod 2 p)$.

(b) $n$ is odd, $a_{(n+1) / 2}$ is even, and $\left(a_{1}, \ldots, a_{n}\right)$ is symmetric.

Before proving Theorem II.3.2, we describe the elements of $\operatorname{Aut}(\mathcal{D})$. Let $\widetilde{X}$ and $\tilde{Y}$ be the homeomorphisms of $\left(\mathbb{R}^{2}, \mathbb{Z}^{2}\right) \times I$ defined by

$$
\widetilde{X}((x, y), t)=((x+1, y), t), \quad \tilde{Y}((x, y), t)=((x, y+1), t)
$$

Then both $\widetilde{X}$ and $\widetilde{Y}$ are $\Gamma$-equivariant, and they are compatible with the relations $\dot{\sim}, \sim_{r}$, and $\sim_{r^{\prime}}$. Hence $\widetilde{X}$ and $\widetilde{Y}$ induce orientation-preserving automorphisms of $\mathcal{D}$, which we denote by $X$ and $Y$ respectively. We can easily see the following.

(1) The group generated by $X$ and $Y$ is isomorphic to $\mathbb{Z}_{2} \oplus \mathbb{Z}_{2}$.

(2) For each $i(2 \leq i \leq c-2)$, both $X$ and $Y$ preserve $\sigma(i)$. The group $\langle X, Y\rangle$ interchanges the tetrahedra of $\sigma(i)$.

Suppose $q^{2} \equiv \pm 1(\bmod p)$. Then, by Lemma II.3.3, the diagram $\Im$ has the 2-fold symmetry as illustrated in Figure II.3.4. This symmetry is realized by the matrix $J$ defined by $J=A^{-1} J_{0} A$, where $A$ and $J_{0}$ are the elements of $G L(2, \mathbb{Z})$ which satisfy the following conditions. [In the following, $\left\{s_{i}\right\}$ denote the vertices of $\Im$ as illustrated in Figure II.3.4.]

CASE 1. The condition in Lemma II.3.3 (1) is satisfied. Then $\widehat{A}\left(s_{1}\right)=1 / 0$, $\widehat{A}\left(s_{2}\right)=0 / 1$, and $J_{0}=\left(\begin{array}{cc}0 & -1 \\ 1 & 0\end{array}\right)$.

CASE 2. The condition in Lemma II.3.3 (2) is satisfied. Then $\widehat{A}\left(s_{1}\right)=1 / 1$, $\widehat{A}\left(s_{2}\right)=1 / 0, \widehat{A}\left(s_{3}\right)=0 / 1$, and $J_{0}=\left(\begin{array}{ll}0 & 1 \\ 1 & 0\end{array}\right)$.

CASE 3. The condition in Lemma II.3.3 (3) is satisfied. Then $\widehat{A}\left(s_{1}\right)=1 / 0$, $\widehat{A}\left(s_{2}\right)=0 / 1$, and $J_{0}=\left(\begin{array}{cc}-1 & 0 \\ 0 & 1\end{array}\right)$. 


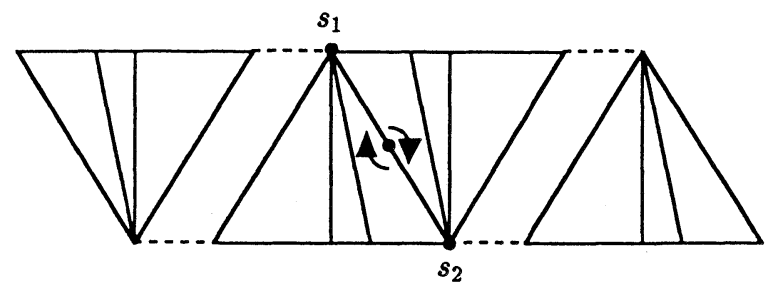

$n:$ even

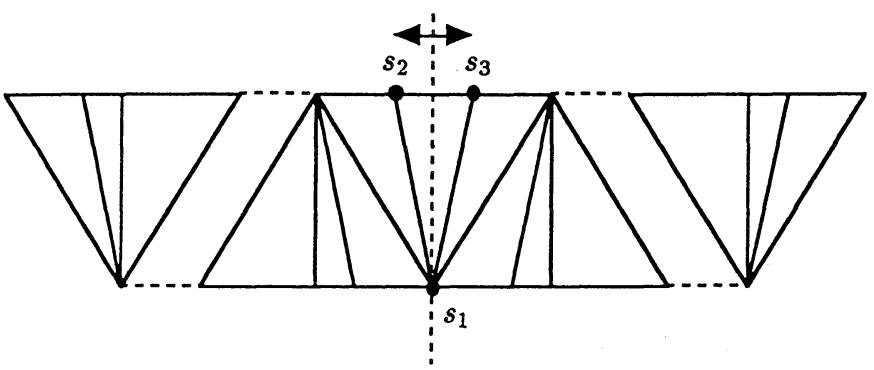

$n:$ odd

$a_{(n+1) / 2}:$ odd

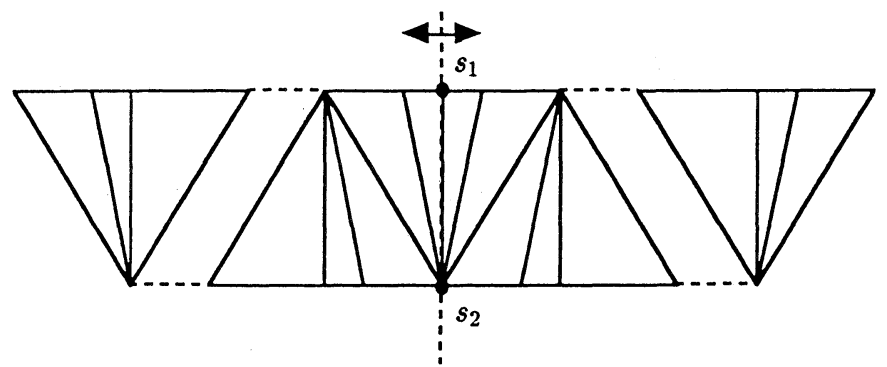

$n:$ odd

$a_{(n+1) / 2}:$ even

Figure II.3.4

Let $\widetilde{Z}$ be the homeomorphism of $\left(\mathbb{R}^{2}, \mathbb{Z}^{2}\right) \times I$ defined by $\widetilde{Z}((x, y), t)=(J(x, y)$, $1-t)$. Then $\widetilde{Z}$ is $\Gamma$-equivariant and is compatible with the relations $\dot{\sim}, \sim_{r}$ and $\sim_{r^{\prime}}$. Hence $\widetilde{Z}$ induces an automorphism of $\mathcal{D}$, which we denote by $Z$. We can see the following:

(1) $Z$ is orientation-reversing in Case 1, and it is orientation-preserving in Cases 2 and 3 .

(2) The subgroup of $\operatorname{Aut}(\mathcal{D})$ generated by $X, Y$, and $Z$ is isomorphic to $D_{4}$ in Cases 1 and 2. It is isomorphic to $\mathbb{Z}_{2} \oplus \mathbb{Z}_{2} \oplus \mathbb{Z}_{2}$ in Case 3 .

We now prove Theorem II.3.2. Let $f$ be an element of $\operatorname{Aut}(\mathcal{D})$. By the proof of Theorem 3.1, $f$ sends $\tau(2)$ to either $\tau(2)$ or $\tau(c-1)$. Suppose $f(\tau(2))=\tau(2)$. Then $f(\sigma(3))=\sigma(3)$. By composing an element of $\langle X, Y\rangle$ if necessary, we may suppose $f$ preserves each of the tetrahedra of $\sigma(2)$ and each of the faces of $\tau(2)$. 
Note that the set of the edges of the faces $\tau(2)$ is equal to the union of $\varepsilon(2)=\varepsilon\left(a_{1}\right)$ and $\varepsilon(3)$. From the proof of Theorem II.3.1, we see no automorphism of $\mathcal{D}$ can send $\varepsilon(2)$ to $\varepsilon(3)$. By using this fact, we can see that $f$ is the identity on $\tau(2)$ and $\sigma(2)$, so $f$ is the identity. Hence, the original $f$ is an element of $\langle X, Y\rangle$. Suppose $f(\tau(2))=\tau(c-1)$. Then by the proof of Theorem II.3.1, the sequence $a_{1}, a_{2}, \ldots, a_{n}$ is symmetric. Hence the automorphism $Z$ exists. By composing $Z$, we can reduce this case to the case $f(\tau(2))=\tau(2)$. This completes the proof of Theorem II.3.2.

At the end of this section we give a proof of Lemma II.3.3 following the arguments of [HK, Lemma 2] and [Si]. To do this, we need the following sublemma, which can be easily proved by induction.

SublemmA II.3.4. Let $a_{1}, \ldots, a_{n}$ be a sequence of positive integers such that $a_{1} \geq 2$ and $a_{n} \geq 2$, and put

$$
\left(\begin{array}{cc}
p & r \\
q & s
\end{array}\right)=\left(\begin{array}{cc}
a_{1} & 1 \\
1 & 0
\end{array}\right)\left(\begin{array}{cc}
a_{2} & 1 \\
1 & 0
\end{array}\right) \cdots\left(\begin{array}{cc}
a_{n} & 1 \\
1 & 0
\end{array}\right)
$$

Then the following holds:

(1) $p s-q r=(-1)^{n}$.

(2) $p / 2 \geq q \geq s, p / 2 \geq r \geq s$.

(3) $q / p=\left[a_{1}, a_{2}, \ldots, a_{n}\right], r / p=\left[a_{n}, a_{n-1}, \ldots, a_{1}\right], s / r=\left[a_{1}, a_{2}, \ldots, a_{n-1}\right]$, and $s / q=\left[a_{n}, a_{n-1}, \ldots, a_{2}\right]$.

Conversely, let $p$ and $q$ be positive integers such that $p / 2 \geq q$. Then there are unique positive integers $r$ and $s$ satisfying the inequalities in (2) such that $p s-q r= \pm 1$. Further, the matrix $\left(\begin{array}{ll}p & r \\ q & s\end{array}\right)$ can be expressed in a unique way as a product $\left(\begin{array}{cc}a_{1} & 1 \\ 1 & 0\end{array}\right)\left(\begin{array}{cc}a_{2} & 1 \\ 1 & 0\end{array}\right) \cdots\left(\begin{array}{cc}a_{n} & 1 \\ 1 & 0\end{array}\right)$, where $a_{1} \geq 2, a_{i} \geq 1(2 \leq i \leq n-1)$, and $a_{n} \geq 2$.

Proof of Lemma II.3.3. First, we prove that Condition (b) implies Condition (a) in each of the three pairs. Suppose $a_{i}=a_{n+1-i}(1 \leq i \leq n)$. Then, by the above sublemma, we see $\left(\begin{array}{cc}p & r \\ q & s\end{array}\right)=\left(\begin{array}{ll}p & q \\ r & s\end{array}\right)$. Hence, we have $q=s$. Since $p s-q r=(-1)^{n}$, we have $q^{2}=(-1)^{n+1}+p s$. Thus we obtain the desired implication in (1) from this identity where $n$ is even. To prove the implications in (2) and (3), assume $n$ is odd, and put $a^{*}=a_{(n+1) / 2}$. Then we obtain the desired implications by the following claim.

ClAIM. If $p$ is odd, then $a^{*} \equiv 1(\bmod 2)$. If $p$ is even, then $a^{*} \equiv s(\bmod 2)$.

Proof of Claim Put $\left(\begin{array}{ll}\alpha & \beta \\ \gamma & \delta\end{array}\right)=\left(\begin{array}{cc}a_{1} & 1 \\ 1 & 0\end{array}\right) \cdots\left(\begin{array}{cc}a_{(n-1) / 2} & 1 \\ 1 & 0\end{array}\right)$. Then $\left(\begin{array}{ll}p & r \\ q & s\end{array}\right)=\left(\begin{array}{cc}\alpha & \beta \\ \gamma & \delta\end{array}\right)\left(\begin{array}{cc}1 & a^{*} \\ 0 & 1\end{array}\right)\left(\begin{array}{cc}\alpha & \gamma \\ \beta & \delta\end{array}\right)$. Hence, We have (i) $p=a^{*} \alpha^{2}+2 \alpha \beta \equiv$ 
$a^{*} \alpha^{2}(\bmod 2)$ and (ii) $s=a^{*} \gamma^{2}+2 \delta \gamma \equiv a^{*} \gamma^{2}(\bmod 2)$. So, if $p$ is odd, then $a^{*} \equiv 1$ $(\bmod 2)$ by (i). Suppose $p$ is even. If $a^{*} \equiv 0(\bmod 2)$, then $s \equiv 0(\bmod 2)$ by (ii). If $a^{*} \equiv 1(\bmod 2)$, then $\alpha \equiv 0(\bmod 2)$ by $(\mathrm{i})$. Since $\alpha \delta-\beta \gamma= \pm 1$, we have $\gamma \equiv 1$ $(\bmod 2)$. Hence $s \equiv 1(\bmod 2)$. This completes the proof of the Claim.

Next, we prove that Condition (a) implies Condition (b) in each of the three pairs. Suppose $q^{2} \equiv \epsilon(\bmod p)$, where $\epsilon= \pm 1$. Let $s$ be the integer such that $p s-q^{2}=\epsilon$. Since $p \geq 2 q$, we see $1 \leq s \leq q$. Hence by Sublemma II.3.4, we see $\left(\begin{array}{ll}p & q \\ q & s\end{array}\right)=\left(\begin{array}{cc}a_{1} & 1 \\ 1 & 0\end{array}\right) \cdots\left(\begin{array}{cc}a_{n} & 1 \\ 1 & 0\end{array}\right)$, where $a_{1}, \ldots, a_{n}$ are positive integers such that $a_{1} \geq 2, a_{i} \geq 1(2 \leq i \leq n-1)$, and $a_{n} \geq 2$. By taking the transpose, we obtain $\left(\begin{array}{cc}p & q \\ q & s\end{array}\right)=\left(\begin{array}{cc}a_{n} & 1 \\ 1 & 0\end{array}\right) \cdots\left(\begin{array}{cc}a_{1} & 1 \\ 1 & 0\end{array}\right)$. By the uniqueness of the expansion, we obtain $a_{i}=a_{n+1-i}$. From this fact we obtain the desired implications.

\section{II.4. The induced cusp triangulation}

Identify the strip $\cup_{i=2}^{c-1} T_{i}$ with the rectangle $I \times I$ in $\mathbb{R}^{2}$, so that the edges $\overline{r_{2} r_{3}}$ and $\overline{r_{2}^{\prime} r_{3}^{\prime}}$, respectively, correspond to $0 \times I$ and $1 \times I$. This gives a triangulation of $I \times I$. Let $G$ be the group of translations of $\mathbb{R}^{2}$ generated by the reflection across $\mathbb{R} \times n(n \in \mathbb{Z})$ and $\pi$-rotations about the vertices $r_{2}$ and $r_{2}^{\prime}$. Then $I \times I$ is a fundamental region for $G$. Thus the above triangulation of $I \times I$ determines a $G$-invariant triangulation of $\mathbb{R}^{2}$, which we denote by $\widetilde{\mathcal{L}}^{\prime}$. Let $\widetilde{\mathcal{L}}$ be the $G$-invariant triangulation of $\mathbb{R}^{2}$ obtained from $\widetilde{\mathcal{L}^{\prime}}$ by deleting the interiors of the translates of the edges $\overline{r_{1} r_{2}}$ and $\overline{r_{1}^{\prime} r_{2}^{\prime}}$. (See Figure II.4.1, where the first coordinate is magnified by the scale 2. See also Figure II.4.2, which is obtained by using SnapPea.) Let $m$ and $\ell$ be the elements of $G$ defined by

$$
\begin{aligned}
& m(x, y)=(x, y+2), \\
& \ell(x, y)= \begin{cases}(x+4, y) & \text { if } p \text { is odd } \\
(x+2, y) & \text { if } p \text { is even. }\end{cases}
\end{aligned}
$$

Let $\mathcal{L}$ be the triangulation of the torus $\mathbb{R}^{2} /\langle m, \ell\rangle$ induced by $\widetilde{\mathcal{L}}$.

THEOREM II.4.1. $\quad \mathcal{L}$ is the triangulation of the peripheral torus (or each of the components of the peripheral tori in case $p$ is even) of $S^{3}-K(p, q)$ induced by the ideal triangulation $\mathcal{D}$.

Proof. For each $i(1 \leq i \leq c-1)$, let $e_{i}$ be the common edge of $T_{i}$ and $T_{i+1}$. For each $i(2 \leq i \leq c-1)$, let $f_{i}$ be the edge of $\Im$ determined by $f_{i}=$ $\operatorname{cl}\left(\partial T_{i}-\left(e_{i} \cup e_{i+1}\right)\right)$. First, we look at a cross section around the ideal vertex $p \dot{\times} I$ in $\widehat{\mathcal{D}}$, where $p$ is a point of $P$. Let $\widetilde{p} \in \mathbb{Z}^{2}$ be a lift of $p$. For each $i(2 \leq i \leq c-2)$, let $\widehat{\mathcal{D}}_{i}$ be the image of the layer of tetrahedra $\widetilde{\mathcal{D}}_{i}$ in $\widehat{\mathcal{D}}$. Truncation yields four boundary triangles around the vertex $\widetilde{p} \dot{\times} I$ in $\widetilde{\mathcal{D}}_{i}$, and hence, it yields two triangles around the vertex $p \dot{\times} I$ in $\widehat{\mathcal{D}}_{i}$ (see Figure II.4.3 (b)). The vertices of these two boundary triangles 

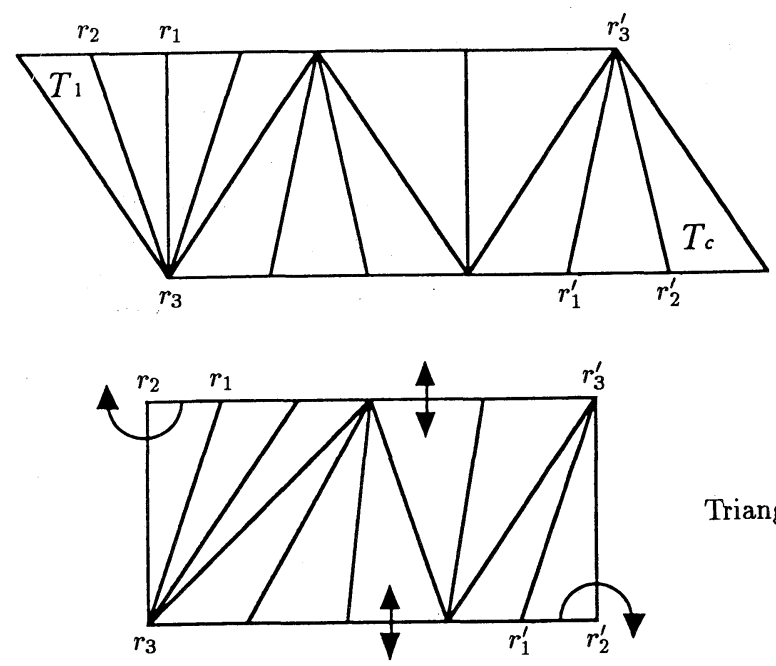

Triangulation of $I \times I$

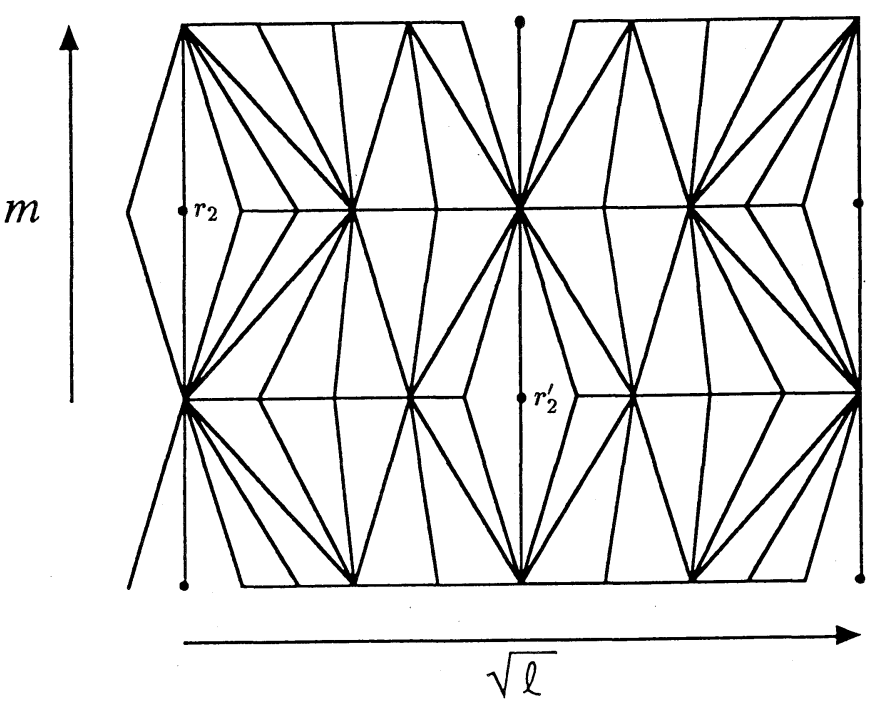

$\tilde{\mathcal{L}}$

Figure II.4.1

correspond to edges of ideal tetrahedra of $\widehat{\mathcal{D}}_{i}$, so each of them has associated to it a slope in $\mathbb{Q} \cup\{1 / 0\}$. We see that the slopes of the vertices of one boundary triangle form the vertices of $T_{i}$ and that the slopes of the vertices of the other boundary triangles form the vertices of $T_{i+1}$ (see Figure II.4.3 (a), (b)). Thus we identify the former boundary triangle with a copy of $T_{i}$ and denote it by $T_{i}(i)$, and we identify the latter boundary triangle with a copy of $T_{i+1}$ and denote it by $T_{i+1}(i)$. Then 


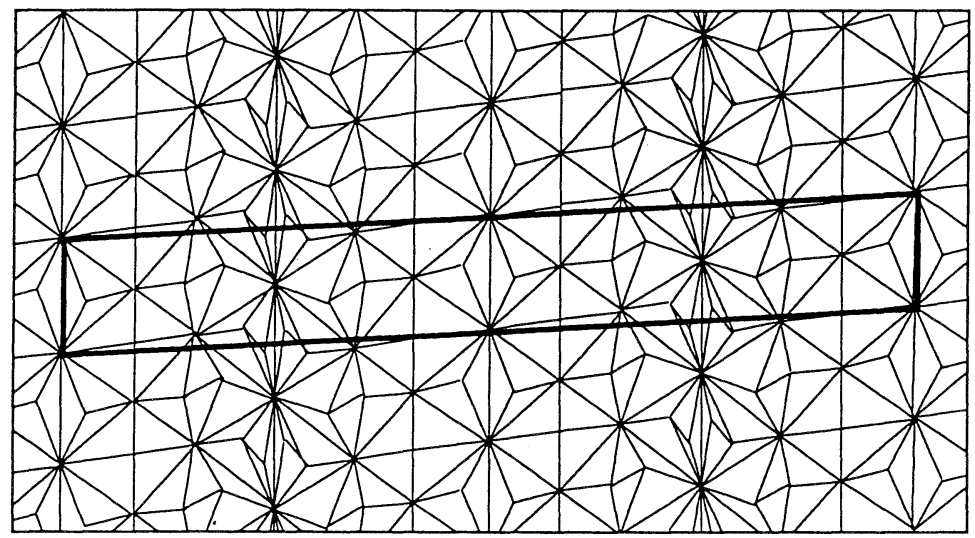

Figure II.4.2

the link of $p \dot{\times} I$ in $\widehat{\mathcal{D}}$ consists of the triangles $\left\{T_{i}(i), T_{i+1}(i) \mid 2 \leq i \leq c-2\right\}$. As illustrated in Figure II.4.3 (c), these triangles are glued together by the following rule.

$$
\begin{aligned}
T_{i}(i) \supset e_{i}(i) & \leftrightarrow e_{i}(i+1) \subset T_{i+1}(i+1) \\
T_{i+1}(i) \supset e_{i+1}(i) & \leftrightarrow e_{i+1}(i+1) \subset T_{i+2}(i+1) \\
T_{i+1}(i) \supset f_{i+1}(i) & \leftrightarrow f_{i+1}(i+1) \subset T_{i+1}(i+1)
\end{aligned}
$$

Here $e_{i}(i)$, for example, denotes the copy of the edge $e_{i}$ in $T_{i}(i)$. By the first relation, we see the union $\bigcup_{i=2}^{c-2} T_{i}(i)$ forms a strip which is homeomorphic to the substrip $\bigcup_{i=2}^{c-2} T_{i}$ of $\Im$. By the second relation, we see the union $\bigcup_{i=2}^{c-2} T_{i+1}(i)$ forms a strip which is homeomorphic to the substrip $\bigcup_{i=3}^{c-1} T_{i}$ of $\Im$. The last relation indicates the way how these two strips are glued together, and we see that the link of $p \dot{\times} I$ in $\widehat{\mathcal{D}}$ is obtained from the restriction of $\widetilde{\mathcal{L}}^{\prime}$ to $I \times[0,2]$ by deleting $T_{c-1}$ and $g\left(T_{2}\right)$, where $g$ is the reflection across $\mathbb{R} \times 1$, and by identifying $(x, 0)$ with $m(x, 0)=(x, 2)$. Here, $x$ varies over the points in $I$ such that both $(x, 0)$ and $m(x, 0)$ are contained in $\operatorname{cl}\left(I \times[0,2]-\left(T_{c-1} \cup g\left(T_{2}\right)\right)\right)$ (see Figure II.4.4 (a), (b)).

Let $\widehat{\mathcal{L}}(i, j)$ be the link of $p \dot{\times} I$ in $\widehat{\mathcal{D}}$, where $p$ is the image of an integral point $(i, j)$. Then the induced triangulation of the boundary tori is obtained from the disjoint union of $\widehat{\mathcal{L}}(i, j)(0 \leq i, j \leq 1)$ through suitable identifications of edges, that is caused by the relations $\sim_{r}$ and $\sim_{r^{\prime}}$ on $\Delta_{2}$ and $\Delta_{c-1}$ respectively. This identification is described as follows. Suppose $r=b / a$ and $r^{\prime}=b^{\prime} / a^{\prime}$, where $a$ and $b$ [resp. $a^{\prime}$ and $b^{\prime}$ ] are relatively prime integers. By the definition of the slopes $r$ and $r^{\prime}$, we see $(a, b)=(2,1) \equiv(0,1)(\bmod 2)$ and $\left(a^{\prime}, b^{\prime}\right) \equiv(p, q)(\bmod 2)$. Then the identification is as follows: 
(a)

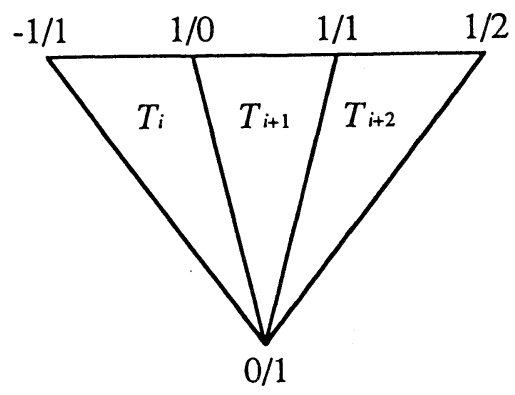

(b)

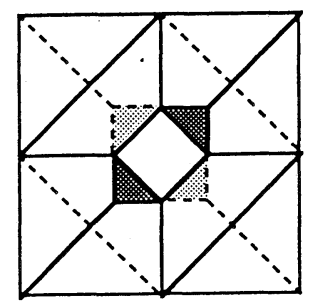

Truncation of a vertex of $\widetilde{D}_{i}$

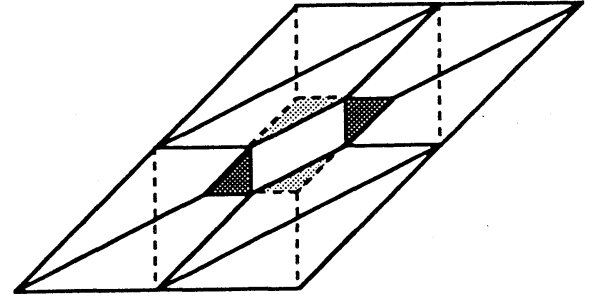

Truncation of a vertex of $\tilde{D}_{i+1}$

(c)

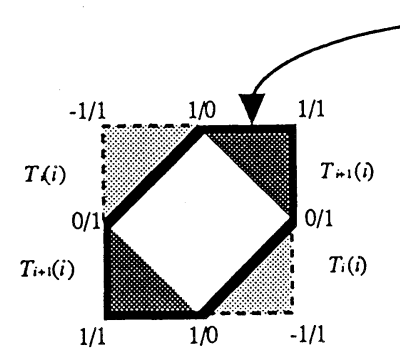

Four boundary triangles in the truncation of $\widetilde{D}_{i}$

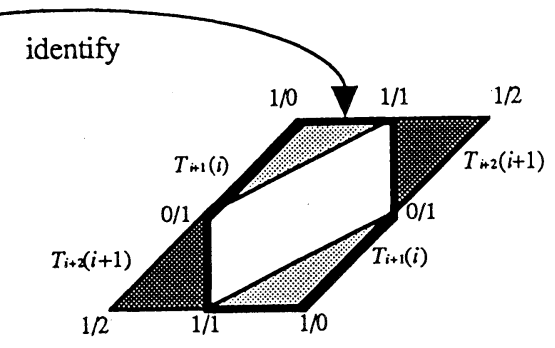

Four boundary triangles in the truncation of $\widetilde{D}_{i+1}$

Figure II.4.3 
(i)

$$
\begin{aligned}
& \overline{r_{1} r_{2}}(i, j) \sim \overline{r_{1} r_{3}}(i, j) \\
& \overline{r_{1}^{\prime} r_{2}^{\prime}}(i, j) \sim \overline{r_{1}^{\prime} r_{3}^{\prime}}(i, j) \\
& \overline{r_{2} r_{3}}(i, j) \sim \overline{r_{3} r_{2}}(i+a, j+b) \\
& \overline{r_{2}^{\prime} r_{3}^{\prime}}(i, j) \sim \overline{r_{3}^{\prime} r_{2}^{\prime}}\left(i+a^{\prime}, j+b^{\prime}\right) .
\end{aligned}
$$

Here $\overline{r_{1} r_{2}}(i, j)$, for example, denotes the copy of the edge $\overline{r_{1} r_{2}}$ in $\widehat{\mathcal{L}}(i, j)$. This can be seen from Figure II.4.5

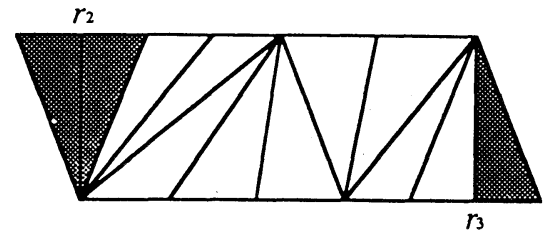

$\bigcup_{i=2}^{c-1} T_{i}(i)$

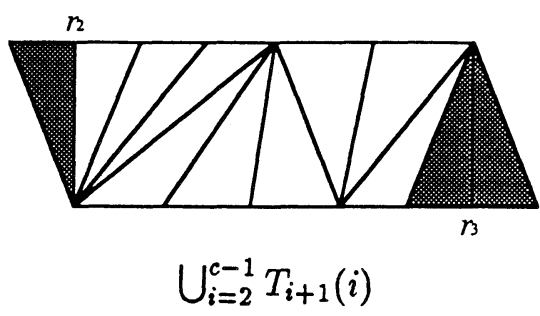

(a)

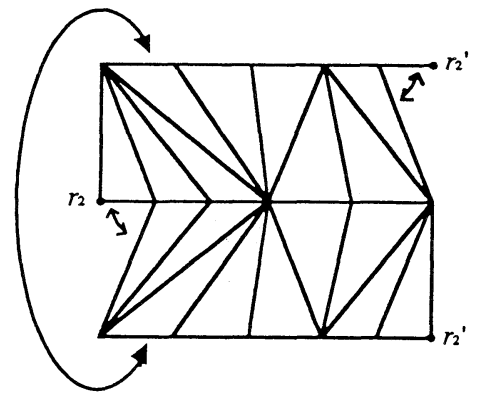

(b)

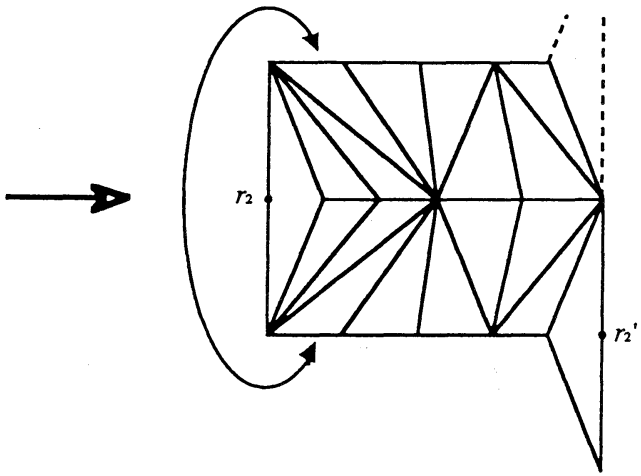

(c)

Figure II.4.4 


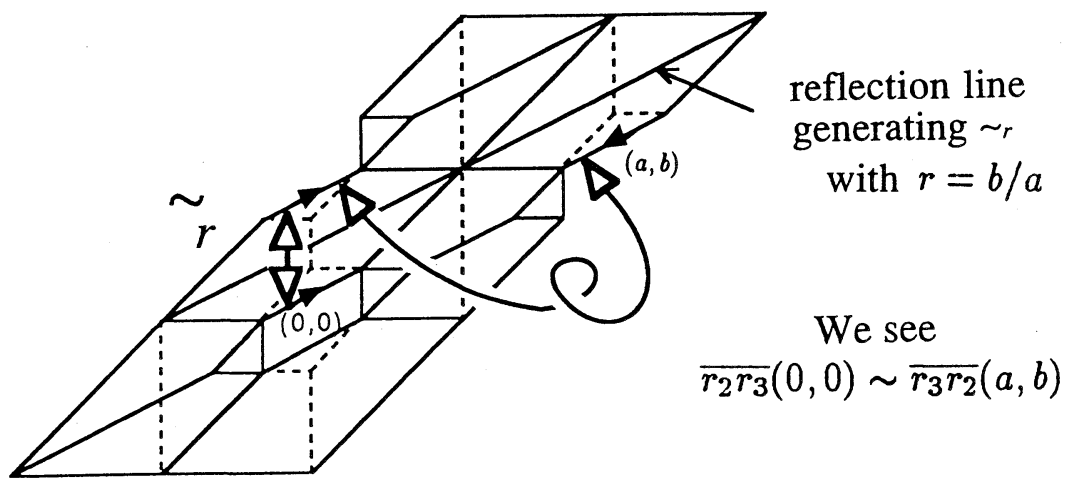

Figure II.4.5

Let $\widehat{\mathcal{L}}^{\prime}(i, j)$ be the space obtained from $\widehat{\mathcal{L}}(i, j)$ by the identifications (i) and (ii). Then it is isomorphic to the quotient of $\left.\widetilde{\mathcal{L}}\right|_{I \times \mathbb{R}}$ by the action of $\langle m\rangle$ (see Figure II.4.4 (c)). The identifications (iii) and (iv) induce the identifications on the four strips $\widehat{\mathcal{L}}^{\prime}(i, j)(0 \leq i, j \leq 1)$ as illustrated in Figure II.4.6.

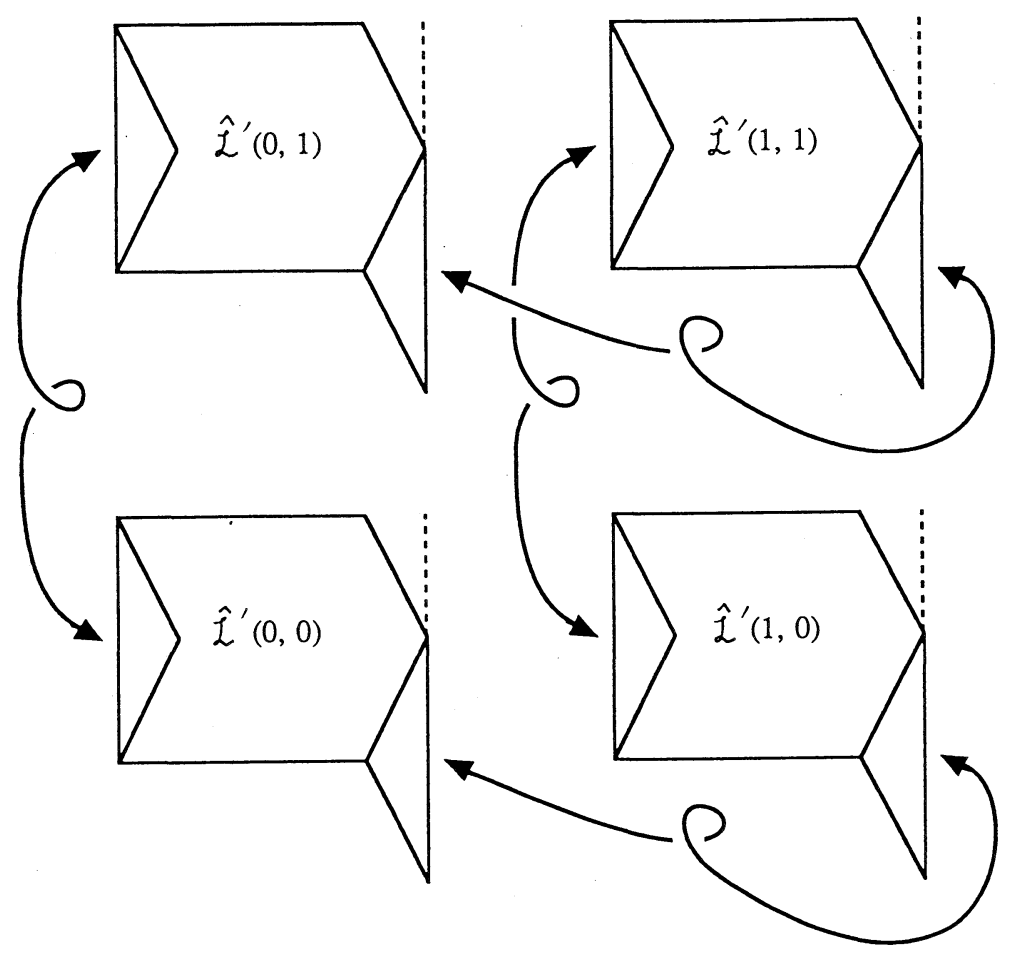

Figure II.4.6 
Thus their developing images in the universal cover of the peripheral torus is obtained from $\left.\widetilde{\mathcal{L}}\right|_{I \times \mathbb{R}}$ by adding its images by the $\pi$-rotations about the points corresponding to (the old) $r_{2}$ and $r_{2}^{\prime}$. This completes the proof of Theorem II.4.1.

\section{II.5 Hyperbolicity equations}

By identifying each topological ideal tetrahedron of $\mathcal{D}$ with a hyperbolic ideal tetrahedron, we obtain a hyperbolic structure on the complement of the 1-skeleton of $\mathcal{D}$. For the structure to give the complete hyperbolic structure of $S^{3}-K(p, q)$, complex parameters of hyperbolic ideal tetrahedra must satisfy the "edge relations" and the "holonomy conditions" (see [Th, Chapter 4] or [BP, Section E.6.i]). In this section, we write down these equations and reduce them to essentially a single polynomial equation in one variable. We also discuss its relation to Riley's work [R1].

To do this, we use the $\mathbb{Z}_{2} \oplus \mathbb{Z}_{2}$-symmetry of $\mathcal{D}$. In other words, instead of working directly on $\mathcal{D}$, we actually study hyperbolicity equations for the orbifold $\mathcal{D} /\langle X, Y\rangle$. Recall that $\mathcal{D}$ consists of $(c-3)$ pairs of ideal tetrahedra $\{\sigma(i) \mid 2 \leq$ $i \leq c-2\}$ and that, for each $i(2 \leq i \leq c-2)$, the group $\langle X, Y\rangle$ permutes the tetrahedra of $\sigma(i)$ and the stabilizer of each of the tetrahedra can be realized by a hyperbolic isometry. So, we give the same complex parameters to the tetrahedra of $\sigma(i)$, which we denote by the symbols $w(i, 1), w(i, 2)$, and $w(i, 3)$; these are related to each other by the following relations (see Figure II.5.1):

$$
w(i, j+1)=\frac{w(i, j)-1}{w(i, j)}, \quad w(i, j+2)=\frac{1}{1-w(i, j)} .
$$

Here the second index $j$ is considered modulo 3 .

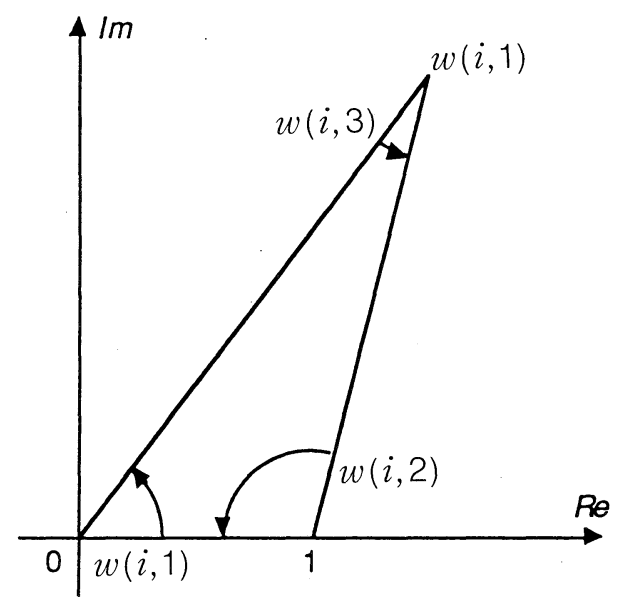

Figure II.5.1 
In the cusp cross section, these parameters are specified as illustrated in Figure II.5.2.

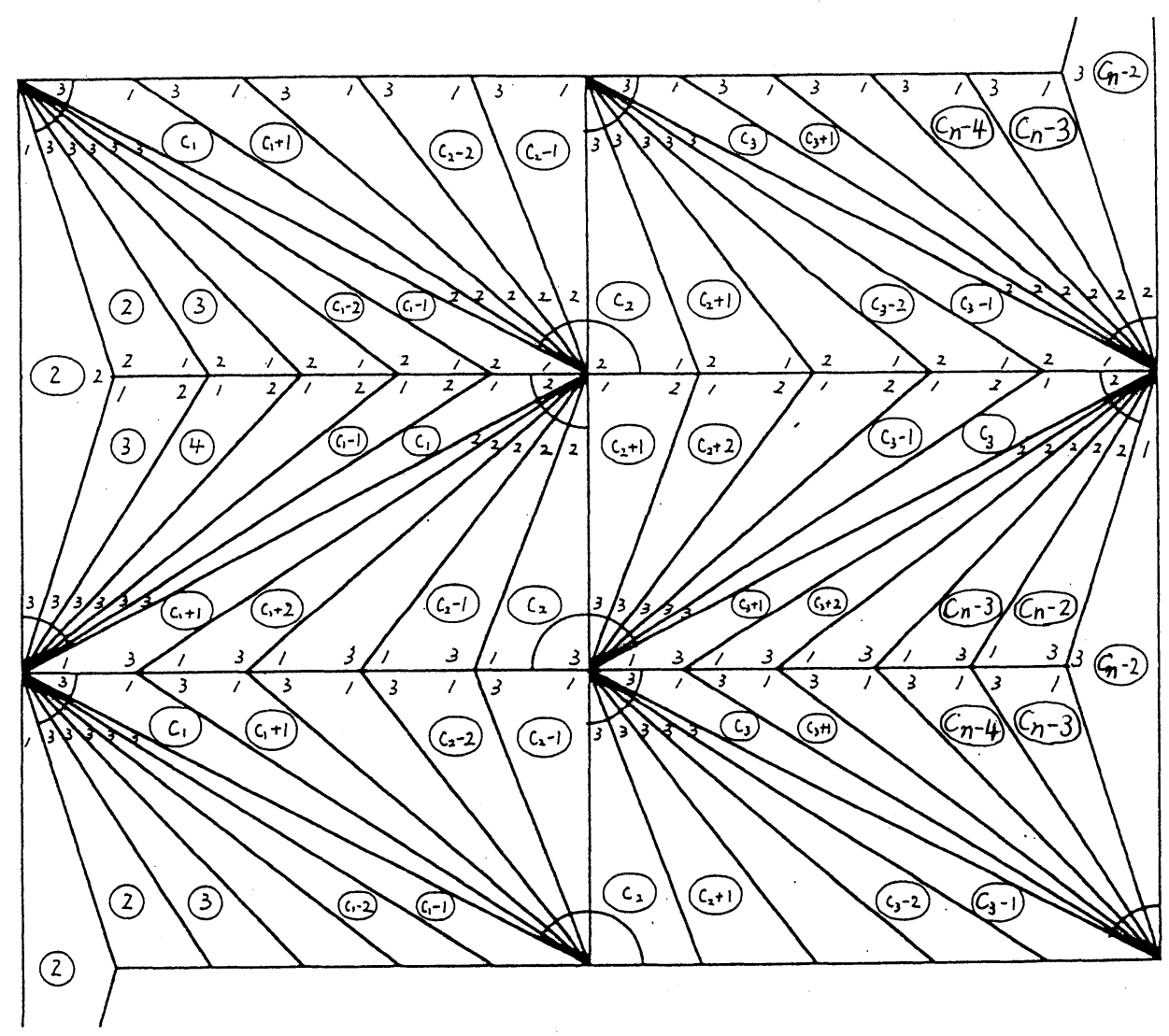

Figure II.5.2 (a) $n$ : even $(=4)$

Note that for each $i(2 \leq i \leq c-3)$, there is essentially a unique vertex of the induced cusp triangulation, such that the edge relation corresponding to which contains the $(i+1)$-th parameter precisely once and consists only of parameters with indices $\leq i+1$. We call it the $i$-th edge relation. There remain two edge relations. One of them consists of two $(c-2)$-th parameters and one $(c-3)$-th parameter, which we call the $(c-2)$-th edge relation. The other relation is called the $(c-1)$-th edge relation. The holonomy conditions are consequences of the edge relations. This can be seen as follows: Let $\rho$ be the holonomy representation of the cusp fundamental group to $\operatorname{Sim}\left(\mathbb{E}^{2}\right)$, the group of the similarities of the Euclidean plane $\mathbb{E}^{2}$, determined by a solution of the edge relations. Then $\rho$ is equivariant with 
respect to the $\mathbb{Z}_{2} \oplus \mathbb{Z}_{2}$-action. This implies that $\operatorname{Im}(\rho)$ is contained in the isometry $\operatorname{group} \operatorname{Isom}\left(\mathbb{E}^{2}\right)$.

The $i$-th edge relation $(2 \leq i \leq c-1)$ is given as follows, where we put $w(1,1)=1$ and $w(c-1,1)=1$.

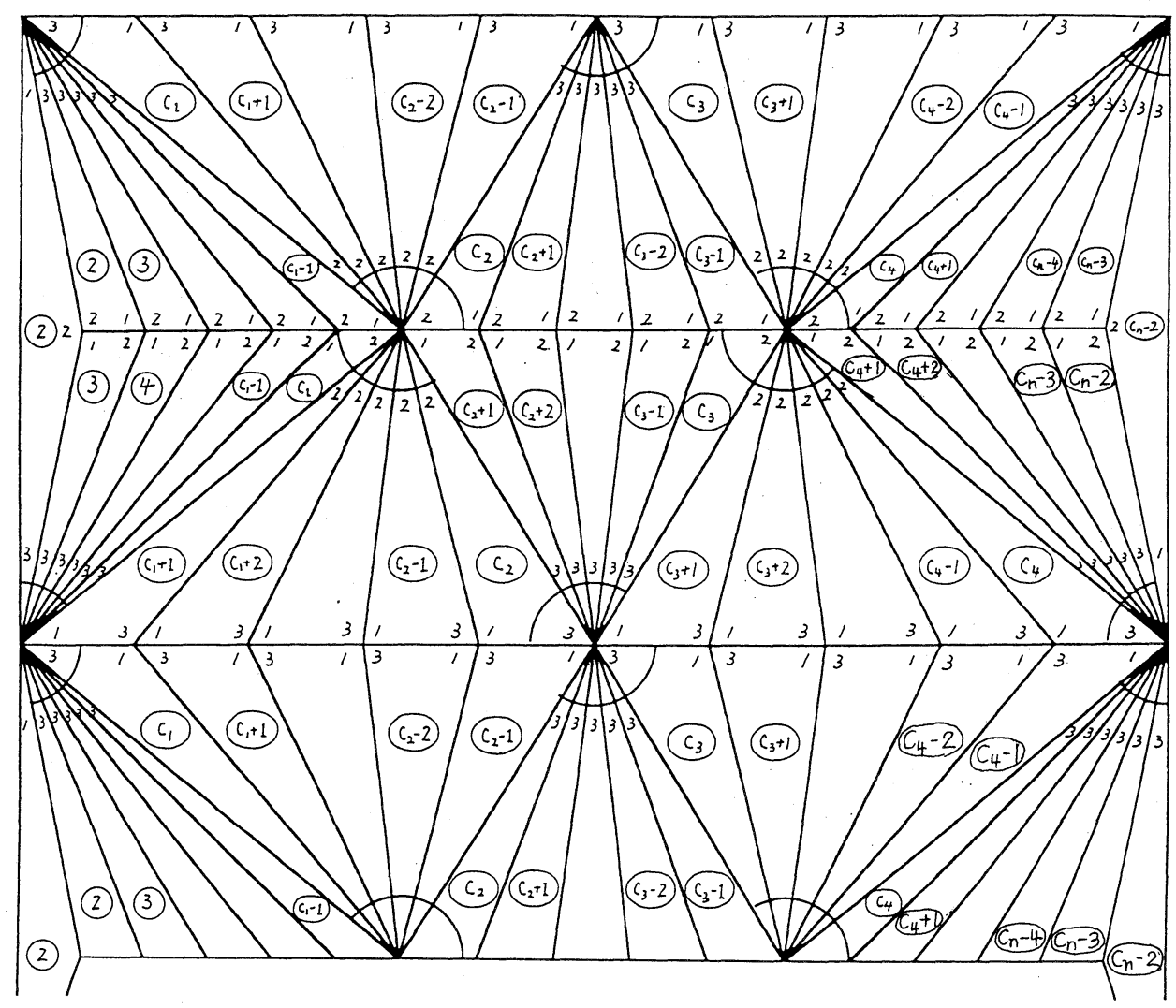

Figure II.5.2 (b) $n$ : odd $(=5)$

CASE 1. $c_{2 r}<i<c_{2 r+1}$ for some $r$. Then

$$
w(i-1,1) w(i, 2)^{2} w(i+1,1)=1 .
$$

CASE 2. $\quad c_{2 r-1}<i<c_{2 r}$ for some $r$. Then

$$
w(i-1,1) w(i, 3)^{2} w(i+1,1)=1 .
$$


CASE 3. $i=c_{1}$. Then

$$
w(2,1)\left\{\prod_{j=2}^{c_{1}} w(j, 3)^{2}\right\} w\left(c_{1}+1,1\right)=-1 .
$$

CASE 4. $i=c_{2 r}$ for some $r$. Then

$$
w\left(c_{2 r-1}-1,1\right)\left\{\prod_{j=c_{2 r-1}}^{c_{2 r}} w(j, 2)^{2}\right\} w\left(c_{2 r}+1,1\right)=1 .
$$

CASE 5. $i=c_{2 r+1}$ for some $r(r \neq 0)$. Then

$$
w\left(c_{2 r}-1,1\right)\left\{\prod_{j=c_{2 r}}^{c_{2 r+1}} w(j, 3)^{2}\right\} w\left(c_{2 r+1}+1,1\right)=1 .
$$

CASE 6. $i=c-1$. Then

$$
\begin{aligned}
& w\left(c_{n-1}-1,1\right)\left\{\prod_{j=c_{n-1}}^{c_{n}-2} w(j, 2)^{2}\right\} w\left(c_{n}-2,1\right)=-1 \text { if } n \text { is even, } \\
& w\left(c_{n-1}-1,1\right)\left\{\prod_{j=c_{n-1}}^{c_{n}-2} w(j, 3)^{2}\right\} w\left(c_{n}-2,1\right)=-1 \text { if } n \text { is odd. }
\end{aligned}
$$

Any solution $\{w(i, 1) \mid 2 \leq i \leq c-2\} \subset \mathbb{C}-\{0,1\}$ of this system of equations determines a parabolic representation of the link group (see [Ta2, Section 6]); it determines the complete hyperbolic structure of the link complement, if and only if $\operatorname{Im}(w(i, 1))>0(2 \leq i \leq c-2)$.

We reduce the above system of equations to a single equation, which is represented by a rational function in one variable. Let $\phi_{i}=\phi_{i}(z)$ be the rational function in one variable $z$ defined by the following recursive formula:

$$
\begin{aligned}
& \phi_{1}=1 \\
& \phi_{2}=z, \\
& \phi_{i+1}= \begin{cases}\frac{1}{\phi_{i-1}}\left(\frac{\phi_{i}}{\phi_{i}-1}\right)^{2} & \text { if } c_{2 r}<i<c_{2 r+1} \text { for some } r, \\
\frac{\left(1-\phi_{i}\right)^{2}}{\phi_{i-1}} & \text { if } c_{2 r-1}<i<c_{2 r} \text { for some } r, \\
\frac{-\phi_{i-1} \phi_{i}}{\left(1-\phi_{i}\right)^{2}} & \text { if } i=c_{2 r} \text { for some } r \\
\frac{-\phi_{i-1}\left(1-\phi_{i}\right)^{2}}{\phi_{i}} & \text { if } i=c_{2 r+1} \text { for some } r .\end{cases}
\end{aligned}
$$


We call the formula for $\phi_{i+1}$ the $i$-th recursive formula.

Proposition II.5.1. The system of hyperbolicity equations for $\mathcal{D}$ (considered over $\mathbb{C})$ is equivalent to the following:

$$
\phi_{c-1}(z)=1, \quad \phi_{i}(z) \neq 1 \quad(2 \leq i \leq c-2) .
$$

Further, for each solution $\omega$, we have

$$
\phi_{i}(\omega)=w(i, 1) \quad(2 \leq i \leq c-2) .
$$

Proof. Represent the complex parameter $w(2,1)$ by $z$, and put $\phi_{i}=w(i, 1)$ $(1 \leq i \leq c-1)$ anew. Then $\phi_{1}=1$ and $\phi_{c-1}=1$ by the convention. We prove that this new $\phi_{i}$ satisfies the above recursive formula by showing that (1) the $i$-th recursive formula follows from the $i$-th edge relation $(2 \leq i \leq c-2)$, and (2) the $(c-1)$-th edge relation is a consequence of the recursive formulas and the equation $\phi_{c-1}=1$. If $i$ is as in Case 1 or 2, then the $i$-th vertex relation clearly induces the recursive formula for $\phi_{i+1}$. To study Case 3, we need the following claim.

Claim. $\quad \prod_{j=2}^{c_{1}-1} w(j, 3)^{2}=\frac{\phi_{c_{1}}}{\phi_{2} \phi_{c_{1}-1}}$.

Proof. By the $j$-th recursive formula $\left(2 \leq j \leq c_{1}-1\right)$, we see

$$
w(j, 3)^{2}=\frac{1}{\left(1-\phi_{j}\right)^{2}}=\frac{\phi_{j-1} \phi_{j+1}}{\phi_{j}^{2}} \quad\left(2 \leq j \leq c_{1}-1\right) .
$$

Hence, we see

$$
\prod_{j=2}^{c_{1}-1} w(j, 3)^{2}=\prod_{j=2}^{c_{1}-1} \frac{\phi_{j-1} \phi_{j+1}}{\phi_{j}^{2}}=\frac{\phi_{1} \phi_{c_{1}}}{\phi_{2} \phi_{c_{1}-1}}=\frac{\phi_{c_{1}}}{\phi_{2} \phi_{c_{1}-1}} .
$$

By using the above claim, we can see that the $c_{1}$-th edge relation implies the $c_{1}$-th recursive formula. To treat Cases 4 and 5 , we need the following claim:

Claim. (1) Suppose the $i$-th recursive formula holds for each $i$ less than $c_{2 r}$. Then

$$
w\left(c_{2 r-1}-1,1\right)\left\{\prod_{j=c_{2 r-1}}^{c_{2 r}-1} w(j, 2)^{2}\right\}=\frac{-\phi_{c_{2 r}}}{\phi_{c_{2 r}-1}} .
$$

(2) Suppose the $i$-th recursive formula holds for each $i$ less than $c_{2 r+1}$. Then

$$
w\left(c_{2 r}-1,1\right)\left\{\prod_{j=c_{2 r}}^{c_{2 r_{1}}-1} w(j, 3)^{2}\right\}=\frac{-\phi_{c_{2 r}+1}}{\phi_{c_{2 r+1}-1}} .
$$


PRoOF. (1) By the $c_{2 r-1}$-th recursive formula, we see

$$
w\left(c_{2 r-1}-1,1\right) w\left(c_{2 r-1}, 2\right)^{2}=\phi_{c_{2 r-1}-1} \frac{\left(\phi_{c_{2 r-1}}-1\right)^{2}}{\phi_{c_{2 r-1}}}=-\frac{\phi_{c_{2 r-1}+1}}{\phi_{c_{2 r-1}}} .
$$

Further, by using the $j$-th recursive formula $\left(c_{2 r-1}+1 \leq j \leq c_{2 r}-1\right)$, we see

$$
w(j, 2)^{2}=\left\{\frac{\phi_{j}-1}{\phi_{j}}\right\}^{2}=\frac{\phi_{j-1} \phi_{j+1}}{\phi_{j}^{2}} .
$$

Hence

$$
\prod_{j=c_{2 r-1}+1}^{c_{2 r}-1} w(j, 2)^{2}=\prod_{j=c_{2 r-1}}^{c_{2 r}-1} \frac{\phi_{j-1} \phi_{j+1}}{\phi_{j}^{2}}=\frac{\phi_{c_{2 r-1}} \phi_{c_{2 r}}}{\phi_{c_{2 r-1}+1} \phi_{c_{2 r}-1}}
$$

Hence

$$
w\left(c_{2 r-1}-1,1\right)\left\{\prod_{j=c_{2 r-1}}^{c_{2 r}-1} w(j, 2)^{2}\right\}=-\frac{\phi_{c_{2 r-1}+1}}{\phi_{c_{2 r-1}}} \frac{\phi_{c_{2 r-1}} \phi_{c_{2 r}}}{\phi_{c_{2 r-1}+1} \phi_{c_{2 r}-1}}=\frac{-\phi_{c_{2 r}}}{\phi_{c_{2 r}-1}} .
$$

(2) can be proved similarly.

By using the above claim, we obtain the $i$-th recursive formula when $i$ is as in Case 4 or 5 . We can see by a similar argument that the $(c-1)$-th edge relation is a consequences of the recursive formulas and the equation $\phi_{c-1}=1$. Further, for each root $\omega$ of the equation $\phi_{c-1}(z)=1$, we see $\phi_{i}(\omega)$ belongs to $\mathbb{C}-\{0,1\}$ for any $i(2 \leq i \leq c-2)$, if and only if $\phi_{i}(\omega) \neq 1$ for any $i(2 \leq i \leq c-2)$. This completes the proof of Proposition II.5.1.

To simplify the recursive formula of $\phi_{i}$, we introduce some notations. Let $k$ be a non-negative integer, and let $\mathbf{v}=\left(v_{1}, \ldots, v_{k}\right)$ be an integral vector. Here, if $k=0$, we regard $\mathbf{v}=\emptyset$, and if $k=1$, we identify $\mathbf{v}$ with an integer $v_{1}$. The vector $\left(v_{1}, \ldots, v_{k-1}\right)$ is denoted by $\mathbf{v}_{0}$. For an integer $w$, put

$$
\begin{aligned}
& \mathbf{v}+w=\left(v_{1}, \ldots, v_{k-1}, v_{k}+w\right) \\
& \mathbf{v} \oplus w=\left(v_{1}, \ldots, v_{k}, w\right) .
\end{aligned}
$$

Let $\psi(\mathbf{v})=\psi(\mathbf{v})(z)$ be a rational function in one variable $z$ defined recursively by the following formula.

$$
\begin{aligned}
\psi(0) & =0, \quad \psi(1)=1, \quad \psi(2)=1 / z \\
\psi(\mathbf{v}+1) & =\frac{(\psi(\mathbf{v})-1)^{2}}{\psi(\mathbf{v}-1)} \quad(\mathbf{v} \neq 1), \\
\psi(\mathbf{v} \oplus 0) & =\frac{1}{\psi(\mathbf{v})}, \\
\psi(\mathbf{v} \oplus 1) & =\frac{-\psi(\mathbf{v}+1)}{\psi(\mathbf{v})} \quad\left(=\frac{-(\psi(\mathbf{v})-1)^{2}}{\psi(\mathbf{v}-1) \psi(\mathbf{v})}\right)
\end{aligned}
$$


Put $\mathbf{a}=\left(a_{1}, a_{2}, \ldots, a_{n}\right)$, where $a_{1}, \ldots, a_{n}$ are as in Section II.2. We say that $\mathbf{v}=\left(v_{1}, v_{2}, \ldots, v_{k}\right)$ is a subvector of $\mathbf{a}$, if $k \leq n, v_{i}=a_{i}(1 \leq i \leq k-1)$, and $1 \leq v_{k} \leq a_{k}$. Put $|\mathbf{v}|=\sum_{i=1}^{k} v_{i}$. Then we can easily obtain the following:

Lemma II.5.2. Suppose $\mathbf{v}$ is a subvector of $\mathbf{a}$. Then

$$
\psi(\mathbf{v})= \begin{cases}\phi_{|\mathbf{v}|} & \text { if } \operatorname{dim} \mathbf{v} \equiv 0(\bmod 2), \\ 1 / \phi_{|\mathbf{v}|} & \text { if } \operatorname{dim} \mathbf{v} \equiv 1(\bmod 2) .\end{cases}
$$

To express the rational function $\psi(\mathbf{v})$ as a quotient of polynomials, we introduce some notations. Let $\alpha(\mathbf{v})$ be an integer defined by the following recursive formulas:

$$
\begin{aligned}
\alpha(\emptyset) & =1, \quad \alpha(w)=w \\
\alpha(\mathbf{v} \oplus w) & =w \alpha(\mathbf{v})+\alpha\left(\mathbf{v}_{0}\right)
\end{aligned}
$$

It should be noted that

$$
\frac{\alpha\left(v_{2}, \ldots, v_{k}\right)}{\alpha\left(v_{1}, \ldots, v_{k}\right)}=\left[v_{1}, \ldots, v_{k}\right]
$$

Next, define $\epsilon(\mathbf{v}) \in\{ \pm 1\}$ and $\delta(\mathbf{v}) \in\{0,1\}$ for each integral vector $\mathbf{v}$ by the following recursive formulas:

$$
\begin{aligned}
\epsilon(\emptyset) & =+1, \delta(\emptyset) \equiv 0(\bmod 2) \\
\delta(\mathbf{v}+w) & \equiv \delta(\mathbf{v})+w(\bmod 2), \\
\delta(\mathbf{v} \oplus 0) & \equiv \begin{cases}1(\bmod 2) & \text { if } \epsilon(\mathbf{v})=\epsilon\left(\mathbf{v}_{0}\right)=-1, \\
0(\bmod 2) & \text { otherwise }\end{cases} \\
\epsilon(\mathbf{v}) & = \begin{cases}-1 & \text { if } \epsilon\left(\mathbf{v}_{0}\right)=+1 \\
(-1)^{\delta(\mathbf{v})} & \text { if } \epsilon\left(\mathbf{v}_{0}\right)=-1\end{cases}
\end{aligned}
$$

Finally, let $f(\mathbf{v})=f(\mathbf{v})(z)$ be the integral polynomial in one variable $z$ defined by the following recursive formulas:

$$
\begin{aligned}
f(\emptyset) & =1, f(0)=0, f(1)=1 \\
f(\mathbf{v}+1) & =f\left(\mathbf{v}_{0}\right) f(\mathbf{v})-\epsilon\left(\mathbf{v}_{0}\right) z^{\alpha\left(\mathbf{v}_{0}\right)} f(\mathbf{v}-1), \\
f(\mathbf{v} \oplus 0) & =f\left(\mathbf{v}_{0}\right), \\
f(\mathbf{v} \oplus 1) & =f(\mathbf{v}+1) .
\end{aligned}
$$

Lemma II.5.3. The following identity holds for any $\mathbf{v}(\neq \emptyset)$.

$$
\psi(\mathbf{v})=\epsilon\left(\mathbf{v}_{0}\right)^{\delta(\mathbf{v})} \frac{f(\mathbf{v})^{2}}{z^{\alpha(\mathbf{v}-1)} f\left(\mathbf{v}_{0}\right)^{2}}=\epsilon\left(\mathbf{v}_{0}\right)^{\delta(\mathbf{v})} \frac{z^{\alpha\left(\mathbf{v}_{0}\right)} f(\mathbf{v})^{2}}{z^{\alpha(\mathbf{v})} f\left(\mathbf{v}_{0}\right)^{2}} .
$$


To prove this lemma, we need the following sublemma.

Sublemma II.5.4. The following identity holds for any $\mathbf{v}(\neq \emptyset)$.

$$
f(\mathbf{v})^{2}-\epsilon\left(\mathbf{v}_{0}\right)^{\delta(\mathbf{v})} z^{\alpha(\mathbf{v}-1)} f\left(\mathbf{v}_{0}\right)^{2}=f(\mathbf{v}-1) f(\mathbf{v}+1) .
$$

Proof. $\quad$ Put $f(\mathbf{v}, w)=f(\mathbf{v} \oplus w), \alpha(\mathbf{v}, w)=\alpha(\mathbf{v} \oplus w)$, and $\delta(\mathbf{v}, w)=\delta(\mathbf{v} \oplus w)$. Then the sublemma is equivalent to the following identity:

$$
f(\mathbf{v}, w-1) f(\mathbf{v}, w+1)-f(\mathbf{v}, w)^{2}=-\epsilon(\mathbf{v})^{\delta(\mathbf{v}, w)} z^{\alpha(\mathbf{v}, w-1)} f(\mathbf{v})^{2} .
$$

We prove this identity by induction on the dimension $k$ of $\mathbf{v}$. First, note that the following identity holds by the defining formula of $f$.

$$
\begin{aligned}
\left(\begin{array}{cc}
f(\mathbf{v}, w+1) & f(\mathbf{v}, w) \\
f(\mathbf{v}, w) & f(\mathbf{v}, w-1)
\end{array}\right) & =\left(\begin{array}{cc}
f(\mathbf{v}) & -\epsilon(\mathbf{v}) z^{\alpha(\mathbf{v})} \\
1 & 0
\end{array}\right)\left(\begin{array}{cc}
f(\mathbf{v}, w) & f(\mathbf{v}, w-1) \\
f(\mathbf{v}, w-1) & f(\mathbf{v}, w-2)
\end{array}\right) \\
& =\left(\begin{array}{cc}
f(\mathbf{v}) & -\epsilon(\mathbf{v}) z^{\alpha(\mathbf{v})} \\
1 & 0
\end{array}\right)^{w-1}\left(\begin{array}{ll}
f(\mathbf{v}, 2) & f(\mathbf{v}, 1) \\
f(\mathbf{v}, 1) & f(\mathbf{v}, 0)
\end{array}\right) .
\end{aligned}
$$

By taking the determinants, we obtain

$$
f(\mathbf{v}, w+1) f(\mathbf{v}, w-1)-f(\mathbf{v}, w)^{2}=\epsilon(\mathbf{v})^{w-1} z^{(w-1) \alpha(\mathbf{v})}\left\{f(\mathbf{v}, 2) f(\mathbf{v}, 0)-f(\mathbf{v}, 1)^{2}\right\} .
$$

Hence, (1) is equivalent to the following identity.

$$
f(\mathbf{v}, 2) f(\mathbf{v}, 0)-f(\mathbf{v}, 1)^{2}=-\epsilon(\mathbf{v})^{\delta(\mathbf{v}, w)-w+1} z^{\alpha\left(\mathbf{v}_{0}\right)} f(\mathbf{v})^{2} .
$$

If $k=0$, i.e., $\mathbf{v}=\emptyset$, then we can easily check this identity. Thus the identity (1) holds in case $\operatorname{dim} \mathbf{v}=0$. Suppose (1) holds for any vector of dimension $k$. Let $\mathbf{v}$ be an integral vector of dimension $k+1$. By definition, we have

$$
\begin{aligned}
& f(\mathbf{v}, 0)=f\left(\mathbf{v}_{0}\right) \\
& f(\mathbf{v}, 1)=f(\mathbf{v}+1) \\
& f(\mathbf{v}, 2)=f(\mathbf{v}) f(\mathbf{v}+1)-\epsilon(\mathbf{v}) z^{\alpha(\mathbf{v})} f\left(\mathbf{v}_{0}\right) .
\end{aligned}
$$

Hence we have

$$
\begin{aligned}
& \text { L.H.S. of }(2) \\
& \qquad \begin{array}{l}
=f(\mathbf{v}) f(\mathbf{v}+1) f\left(\mathbf{v}_{0}\right)-\epsilon(\mathbf{v}) z^{\alpha(\mathbf{v})} f\left(\mathbf{v}_{0}\right)^{2}-f(\mathbf{v}+1)^{2} \\
=f(\mathbf{v}+1)\left\{f(\mathbf{v}) f\left(\mathbf{v}_{0}\right)-f(\mathbf{v}+1)\right\}-\epsilon(\mathbf{v}) z^{\alpha(\mathbf{v})} f\left(\mathbf{v}_{0}\right)^{2} \\
=\epsilon\left(\mathbf{v}_{0}\right) z^{\alpha\left(\mathbf{v}_{0}\right)} f(\mathbf{v}+1) f(\mathbf{v}-1)-\epsilon(\mathbf{v}) z^{\alpha(\mathbf{v})} f\left(\mathbf{v}_{0}\right)^{2}
\end{array}
\end{aligned}
$$


On the other hand, we can easily verify $\epsilon\left(\mathbf{v}_{0}\right)=-\epsilon(\mathbf{v})^{\delta(\mathbf{v}, w)-w+1}$. Hence

$$
\begin{aligned}
& \text { L.H.S. of (2)- R.H.S. of (2) } \\
& \qquad \epsilon\left(\mathbf{v}_{0}\right) z^{\alpha\left(\mathbf{v}_{0}\right)}\left\{f(\mathbf{v}+1) f(\mathbf{v}-1)-f(\mathbf{v})^{2}\right\}-\epsilon(\mathbf{v}) z^{\alpha(\mathbf{v})} f\left(\mathbf{v}_{0}\right)^{2} \\
& =\epsilon\left(\mathbf{v}_{0}\right) z^{\alpha\left(\mathbf{v}_{0}\right)}\left\{-\epsilon\left(\mathbf{v}_{0}\right)^{\delta(\mathbf{v})} z^{\alpha(\mathbf{v}-1)} f\left(\mathbf{v}_{0}\right)^{2}\right\}-\epsilon(\mathbf{v}) z^{\alpha(\mathbf{v})} f\left(\mathbf{v}_{0}\right)^{2} \\
& =-\left\{\epsilon\left(\mathbf{v}_{0}\right)^{\delta(\mathbf{v})+1} z^{\alpha(\mathbf{v}-1)+\alpha\left(\mathbf{v}_{0}\right)}+\epsilon(\mathbf{v}) z^{\alpha(\mathbf{v})}\right\} f\left(\mathbf{v}_{0}\right)^{2} .
\end{aligned}
$$

Here, the second identity follows from the inductive hypothesis. On the other hand, we can easily see $\epsilon\left(\mathbf{v}_{0}\right)^{\delta(\mathbf{v})+1}=-\epsilon(\mathbf{v})$ and $\alpha(\mathbf{v})=\alpha(\mathbf{v}-1)+\alpha\left(\mathbf{v}_{0}\right)$. Thus the above is equal to 0 . Hence the identity (2) is verified. This completes the proof of Sublemma II.5.4.

Proof of Lemma II.5.3. If $\mathbf{v}=0$ or 1 , we can easily verify the identity. Assuming that the identity holds for $\mathbf{v}$ and $\mathbf{v}-1$, we prove that the identity holds for $\mathbf{v}+1, \mathbf{v} \oplus 0$, and $\mathbf{v} \oplus 1$. By using the assumption and Sublemma II.5.4, we see

$$
\begin{aligned}
\psi(\mathbf{v})-1 & =\epsilon\left(\mathbf{v}_{0}\right)^{\delta(\mathbf{v})} \frac{f(\mathbf{v})^{2}-\epsilon\left(\mathbf{v}_{0}\right)^{\delta(\mathbf{v})} z^{\alpha(\mathbf{v}-1)} f\left(\mathbf{v}_{0}\right)^{2}}{z^{\alpha(\mathbf{v}-1)} f\left(\mathbf{v}_{0}\right)^{2}} \\
& =\epsilon\left(\mathbf{v}_{0}\right)^{\delta(\mathbf{v})} \frac{f(\mathbf{v}-1) f(\mathbf{v}+1)}{z^{\alpha(\mathbf{v}-1)} f\left(\mathbf{v}_{0}\right)^{2}} .
\end{aligned}
$$

Hence, by using the inductive hypothesis again, we see

$$
\begin{aligned}
\psi(\mathbf{v}+1) & =\frac{(\psi(\mathbf{v})-1)^{2}}{\psi(\mathbf{v}-1)} \\
& =\frac{\{f(\mathbf{v}-1) f(\mathbf{v}+1)\}^{2} /\left\{z^{2 \alpha(\mathbf{v}-1)} f\left(\mathbf{v}_{0}\right)^{4}\right\}}{\epsilon\left(\mathbf{v}_{0}\right)^{\delta(\mathbf{v}-1)} f(\mathbf{v}-1)^{2} /\left\{z^{\alpha(\mathbf{v}-2)} f\left(\mathbf{v}_{0}\right)^{2}\right\}} \\
& =\epsilon\left(\mathbf{v}_{0}\right)^{\delta(\mathbf{v}-1)} \frac{f(\mathbf{v}+1)^{2}}{z^{\{2 \alpha(\mathbf{v}-1)-\alpha(\mathbf{v}-2)\}} f\left(\mathbf{v}_{0}\right)^{2}} \\
& =\epsilon\left(\mathbf{v}_{0}\right)^{\delta(\mathbf{v}+1)} \frac{f(\mathbf{v}+1)^{2}}{z^{\alpha(\mathbf{v})} f\left(\mathbf{v}_{0}\right)^{2}}
\end{aligned}
$$

Thus the identity holds for $\mathbf{v}+1$.

Next, we prove the identity for $(\mathbf{v}, 0)$. By definition and the inductive hypothesis, we see

$$
\begin{aligned}
\psi(\mathbf{v}, 0) & =1 / \psi(\mathbf{v}) \\
& =\epsilon\left(\mathbf{v}_{0}\right)^{\delta(\mathbf{v})} \frac{z^{\alpha(\mathbf{v}-1)} f\left(\mathbf{v}_{0}\right)}{f(\mathbf{v})^{2}}
\end{aligned}
$$

On the other hand, we can see

$$
\begin{aligned}
\epsilon\left(\mathbf{v}_{0}\right)^{\delta(\mathbf{v})} & =\epsilon(\mathbf{v})^{\delta\left(\mathbf{v}_{0}\right)} \\
f\left(\mathbf{v}_{0}\right) & =f(\mathbf{v}, 0), \\
\alpha(\mathbf{v}-1) & =-\alpha(\mathbf{v},-1) .
\end{aligned}
$$


Hence, we can see the identity for $\mathbf{v} \oplus 0=(\mathbf{v}, 0)$, i.e.,

$$
\psi(\mathbf{v}, 0)=\epsilon(\mathbf{v})^{\delta(\mathbf{v}, 0)} \frac{f(\mathbf{v}, 0)^{2}}{z^{\alpha(\mathbf{v},-1)} f(\mathbf{v})^{2}} .
$$

Finally, we prove the identity for $\mathbf{v} \oplus 1=(\mathbf{v}, 1)$. By inductive hypothesis, we have

$$
\begin{aligned}
\psi(\mathbf{v}) & =\epsilon\left(\mathbf{v}_{0}\right)^{\delta(\mathbf{v})} \frac{f(\mathbf{v})^{2}}{z^{\alpha(\mathbf{v}-1) f\left(\mathbf{v}_{0}\right)^{2}}} \\
\psi(\mathbf{v}+1) & =\epsilon\left(\mathbf{v}_{0}\right)^{\delta(\mathbf{v}+1)} \frac{f(\mathbf{v}+1)^{2}}{z^{\alpha(\mathbf{v}) f\left(\mathbf{v}_{0}\right)^{2}}}
\end{aligned}
$$

Hence

$$
\begin{aligned}
\psi(\mathbf{v}, 1) & =\frac{-\psi(\mathbf{v}+1)}{\psi(\mathbf{v})} \\
& =-\epsilon\left(\mathbf{v}_{0}\right) \frac{f(\mathbf{v}+1)^{2}}{z^{\alpha\left(\mathbf{v}_{0}\right)} f(\mathbf{v})^{2}} \\
& =\epsilon(\mathbf{v})^{\delta(\mathbf{v}, 1)} \frac{f(\mathbf{v}+1)^{2}}{z^{\alpha(\mathbf{v}, 0)} f(\mathbf{v})^{2}}
\end{aligned}
$$

This completes the proof of Lemma II.5.3.

Hence, we obtain the following:

THEOREM II.5.5. The system of hyperbolicity equations for $\mathcal{D}\left[a_{1}, \ldots, a_{n}\right]$ is equivalent to the following:

$$
\begin{aligned}
& f(\mathbf{a})(z)=0, \\
& f(\mathbf{v})(z) \neq 0 \quad \text { for any subvector } \mathbf{v} \text { of } \mathbf{a} .
\end{aligned}
$$

For each solution $\omega$ of this equation, the complex parameter $w(i, 1)(2 \leq i \leq c-2)$ is given by

$$
w(i, 1)= \begin{cases}\psi(\mathbf{v})(\omega)=\epsilon\left(\mathbf{v}_{0}\right)^{\delta(\mathbf{v})} \frac{f(\mathbf{v})(\omega)^{2}}{z^{\alpha(\mathbf{v}-1)} f\left(\mathbf{v}_{0}\right)(\omega)^{2}} & \text { if } \operatorname{dim} \mathbf{v} \equiv 0(\bmod 2) \\ 1 / \psi(\mathbf{v})(\omega)=\epsilon\left(\mathbf{v}_{0}\right)^{\delta(\mathbf{v})} \frac{z^{\alpha(\mathbf{v}-1)} f\left(\mathbf{v}_{0}\right)(\omega)^{2}}{f(\mathbf{v})(\omega)^{2}} & \text { if } \operatorname{dim} \mathbf{v} \equiv 1(\bmod 2)\end{cases}
$$

where $\mathbf{v}$ is a subvector of $\mathbf{a}=\left(a_{1}, \ldots, a_{n}\right)$ such that $|\mathbf{v}|=i$. Each solution $\omega$ determines a parabolic representation of the link group to $P S L(2, \mathbb{C})$, and it determines the complete hyperbolic structure of the link complement if and only if $\operatorname{Im}(w(i, 1))>0(2 \leq i \leq c-2)$. 
ExAmple II.5.6. The $5_{2}$ knot: This is the 2-bridge knot $K(7,3)$. We see $3 / 7=$ $[2,3]$ and

$$
f(2,1)=1-z, \quad f(2,2)=1-z+z^{2}, \quad f(2,3)=1-z+2 z^{2}-z^{3} .
$$

The polynomial $f(2,3)$ has a unique root $\omega$ with positive imaginary part. This solution determines the following complex parameters:

$$
\begin{aligned}
& w(2,1)=\omega=0.122561+0.744862 i \\
& w(3,1)=\psi(2,3)(\omega)=\left[(-1) \frac{(1-z)^{2}}{z}\right]_{z=\omega}=-1 / \omega^{2}=1.66236+0.562278 i .
\end{aligned}
$$

Hence, the above complex parameters determine the complete hyperbolic structure of the knot complement. By calculating the tilts (cf. [We, SW]), we can see the decomposition $\mathcal{D}$ is the canonical decomposition.

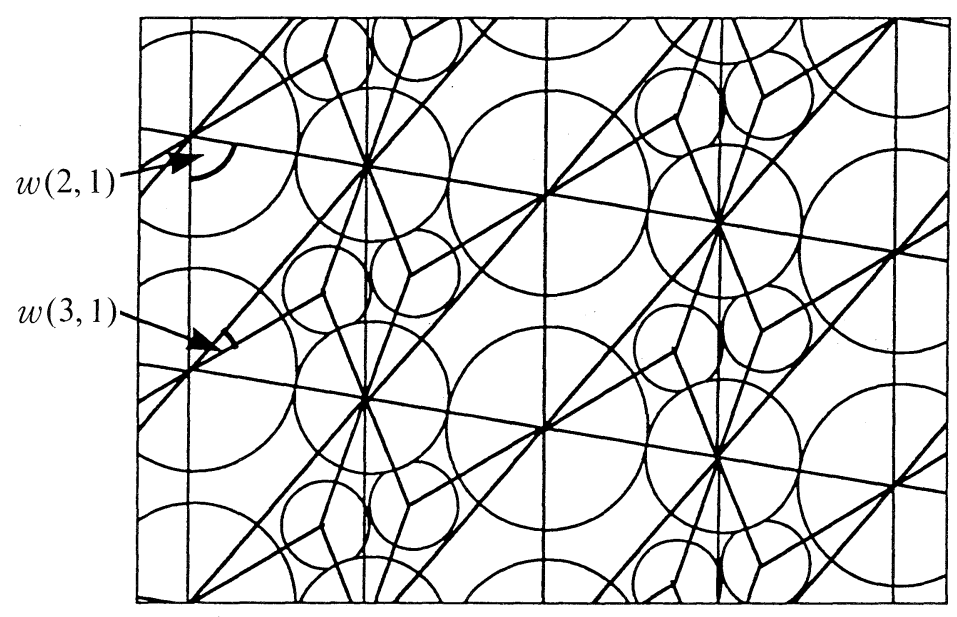

Figure II.5.3

Example II.5.7. The Whitehead link: This is the 2-bridge link $K(8,3)$. We see $3 / 8=[2,1,2]$ and

$$
f(2,1)=1-z, \quad f(2,1,1)=1-z+z^{2}, \quad f(2,1,2)=1-2 z+2 z^{2} .
$$

The complex number $\omega=(1+i) / 2$ is the unique root of $f(2,1,2)$ with positive imaginary part. This solution determines the following complex parameters:

$$
\begin{aligned}
& w(2,1)=\omega=(1+i) / 2 \\
& w(3,1)=\psi(2,1)(\omega)=\left[(-1) \frac{(1-z)^{2}}{z}\right]_{z=\omega}=\omega=(1+i) / 2 .
\end{aligned}
$$


Hence, the above complex parameters determine the complete hyperbolic structure of the link complement. By calculating the tilts, we can see the decomposition $\mathcal{D}$ is the canonical decomposition. It should be noted that $\mathcal{D}$ is a subdivision of the decomposition of the Whitehead link complement into one ideal regular octahedron given by [Th, Section 3.4].

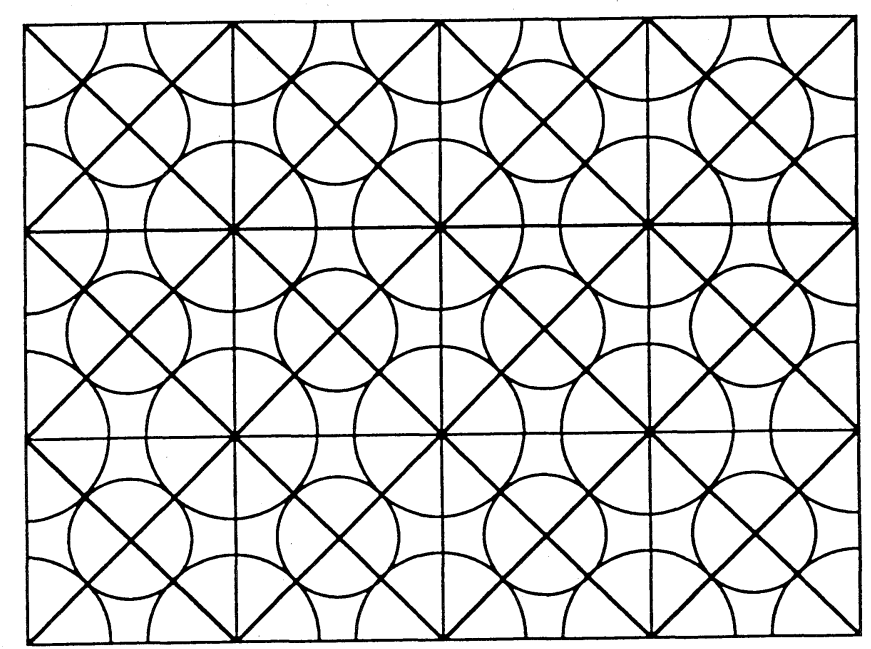

Figure II.5.4

The following lemma, which can be proved easily by induction, gives an estimate of the numbers of the parabolic representations of the 2-bridge knot groups arising from Theorem II.5.5.

LEMMA II.5.8. Let $\mathbf{a}$ be an integral vector without negative entries. If $\alpha(\mathbf{a})$ is odd, then $\operatorname{deg}(f(\mathbf{a}))=(\alpha(\mathbf{a})-1) / 2$. If $\alpha(\mathbf{a})$ is even, then $\operatorname{deg}(f(\mathbf{a})) \leq(\alpha(\mathbf{a})-2) / 2$.

S. Yamada kindly proved the following lemma at our request.

LEMMA II.5.9. (1) The polynomial $f(2, b)(b \geq 2)$ has no repeated roots.

(2) The polynomials $f(2, b)$ and $f\left(2, b^{\prime}\right)\left(b>b^{\prime} \geq 2\right)$ have no common roots.

This lemma says that we can construct precisely $b$ number of parabolic representations of a (non-torus) twist knot (i.e., the 2-bridge knot $K(2 b+1, b)$ with $b \geq 2$ ) by the method of Theorem II.5.5. This reminds us the result of Riley [R1] stating that the knot group of the 2-bridge knot $K(p, q)$ has precisely $(p-1) / 2$ number of parabolic representations. This result leads us to the following conjecture:

Conjecture II.5.10. Let $\mathbf{a}$ be an integral vector without negative entries.

(1) If $\alpha(\mathbf{a})$ is odd, then $f(\mathbf{a})$ has no repeated roots. 
(2) For any subvector $\mathbf{v}$ of $\mathbf{a}, f(\mathbf{v})$ shares no common roots with $f(\mathbf{a})$.

\section{Chapter III. Related topics}

\section{III.1 Relation to alternating diagrams}

In Examples I.2.2 and I.2.3, the edges of the canonical decompositions arise from the crossings of the alternating diagrams. For the canonical decompositions of the links in Example I.2.4 and for the presumably canonical decompositions of the 2-bridge link complements, the arcs arising from the crossings of the alternating diagrams belong to their edge sets. Further, computer experiments using SnapPea tell us that the same results hold for many alternating links. Thus we would like to propose:

CONJECTURE III.1.1. The arc corresponding to each crossing of a reduced alternating diagram of a hyperbolic alternating link is isotopic to an edge of the canonical decomposition of the link complement.

This conjecture is equivalent to the conjecture that the canonical decomposition of a hyperbolic alternating link complement is a certain subdivision of its decomposition into two ideal 3-cells described in Section I.2. We hope this approach sheds new light on the Tait conjecture (which was proved by $[\mathrm{MT}]$ using combinatorial methods) from the point of view of hyperbolic geometry.

\section{III.2 Relation to unknotting tunnels}

An unknotting tunnel for a knot $K$ in $S^{3}$ is an arc, say $\tau$, properly embedded in the knot exterior $E(K)=\operatorname{cl}\left(S^{3}-N(K)\right)$ such that $\operatorname{cl}(E(K)-N(\tau))$ is a handlebody of genus 2. Two unknotting tunnels $\tau_{1}$ and $\tau_{2}$ for $K$ are said to be isotopic [resp. homeomorphic], if there is an ambient isotopy [resp. homeomorphism] of $E(K)$ carrying $\tau_{1}$ to $\tau_{2}$. The unknotting tunnels for the torus knots and the satellite knots were classified by [BRZ] and [MS] respectively. Thus we may restrict our attention to the hyperbolic knots for the study of unknotting tunnels. For the 2bridge knots, it is known that they have six unknotting tunnels in general, and these are classified up to isotopy and homeomorphism (see [K, MS, U]). Further, it is conjectured that these are the only unknotting tunnels for 2-bridge knots (cf. $[\mathrm{Mo}]$ ). To our surprise, these unknotting tunnels constitute very special edges of the triangulations given in the previous chapter. In fact, these are the six edges in $\varepsilon(1)=\varepsilon\left(c_{1}\right)$ and $\varepsilon(c-1)=\varepsilon(c)$ (see Figure II.3.3). Computer experiments using SnapPea tells us that the lengths of these tunnels (i.e., the lengths of the parts of the corresponding geodesics outside fixed cusp neighbourhoods) are short; in particular, one of the the upper or the lower tunnels is the shortest edge. These observations support the results and conjectures presented by Adams [A]. Recently, the list of the knots with $\leq 10$ crossings admitting unknotting tunnels was given by [MSY], and it was observed that if an alternating knot with $\leq 10$ crossings admits an 
unknotting tunnel, then some arc arising from a crossing of its alternating diagram forms an unknotting tunnel. These observations, together with Conjecture III.1.1, seem to support the following conjecture:

CONJECTURE III.2.1. Any unknotting tunnel for a hyperbolic knot is isotopic to an edge of the canonical decomposition.

This conjecture implies the following conjecture:

CONJECTURE III.2.2. Two unknotting tunnels for a knot are isotopic, if and only if they are homotopic.

In fact, Conjecture III.2.1 implies the above conjecture for hyperbolic knots, whereas the results of [BRZ] and [MS] imply it for non-hyperbolic knots.

Acknowledgement. Part of this joint work was done when the first-named author was staying at Toronto University and Ruhr University. He would like to express his hearty thanks to Prof. K. Murasugi and Prof. H. Zieschang for their kind hospitality and encouragement. He would also like to express his hearty thanks to Prof. T. J $\phi$ rgensen for kindly explaining the results of $[\mathrm{J}]$. The second-named author would like to thank the Geometry Center (NSF Science and Technology Research Center for Computation and Visualization of Geometric Structures) for its support. Both authors would like to thank Prof. A. Mednykh for pointing out the overlap of a part of Section I.2 of this paper with a part of [Th, Chapter 6].

\section{References}

[A] C. Adams, Unknotting tunnels in hyperbolic 3-manifolds, Math. Ann., 302 (1995), 177195.

[AHW] C. Adams, M. Hildebrand and J. Weeks, Hyperbolic invariants of knots and links, Trans. A.M.S., 326 (1991), 1-56.

[AR1] I.R. Aitchison and J.H. Rubinstein, Combinatorial cubings, cusps and the dodecahedral knots, Topology 90 (ed. B. Apanasov et al.), (1992) pp. 17-26, Walter de Gruyter.

[AR2] I.R. Aitchison and J.H. Rubinstein, Canonical surgery on alternating link diagrams, Knot 90 (ed. A. Kawauchi), (1992) pp. 543-558, Walter de Gruyter.

[BP] R. Benedetti and C. Petronio, Lectures on hyperbolic geometry, Universitext, Springer, 1992.

[BRZ] M. Boileau, M. Rost and H. Zieschang, On Heegaard decompositions of torus exteriors and related Seifert fibred spaces, Math. Ann., 279 (1988), 553-581.

[BZ] M. Boileau and B. Zimmermann, The $\pi$-orbifold group of a link, Math. Z., 200 (1989), 187-208.

[BS] F. Bonahon and L. Siebenmann, Geometric splittings of knots, and Conway's algebraic knots, preprint.

[C] J.H. Conway, An enumeration of knots and links, Computational problems in abstract algebra (ed. J. Leech), pp. 329-359, Markham, Chicago, 1967.

[EP] D.B.A. Epstein and R.C. Penner, Euclidean decompositions of noncompact hyperbolic 
manifolds, J. Differential Geometry, 27 (1988), 67-80.

[FH] W. Floyd and A. Hatcher, Incompressible surfaces in punctured torus bundles, Topology Appl., 13 (1982), 263-282.

[GL] C. McA. Gordon and J. Luecke, Knots are determined by their complements, J. Amer. Math. Soc., 3 (1989), 371-415.

[HK] R. Hartley and A. Kawauchi, Polynomials of amphicheiral knots, Math. Ann., 243 (1979), 63-70.

[H] A. Hatcher, Hyperbolic structures of arithmetic types on some link complements, J. London Math. Soc., 27 (1983), 345-355.

[HT] A. Hatcher and W. Thurston, Incompressible surfaces in 2-bridge knot complements, Invent. Math., 79 (1985), 225-246.

[HW] S. Henry and J. Weeks, Symmetry groups of hyperbolic knots and links, J. Knot Theory and its ramification, 1 (1992), 185-201.

[J] T. J $\quad$ rgensen, On pairs of punctured tori, in preparation.

[K] T. Kobayashi, A criterion for detecting inequivalent tunnels for a knot, Math. Proc. Cambridge Philos. Soc., 107 (1990), 483-491.

[KS] K. Kodama and M. Sakuma, Symmetry groups of prime knots up to 10 crossings, Knots 90 (ed. A. Kawauchi), pp. 323-340.

$[\mathrm{Me}]$ W. Menasco, Polyhedral representation of surfaces in alternating knots and links, Contemporary Math., 20 (1983), 305-325.

[MT] W. Menasco and M. Thistlethwaite, The classification of alternating links, Ann. Math., 138 (1993), 113-171.

[Mo] K. Morimoto, A note on unknotting tunnels for 2-bridge knots, Bull. Faculty of Eng. Takushoku Univ., 3 (1992), 219-225.

[MS] K. Morimoto and M. Sakuma, On unknotting tunnels for knots, Math. Ann., 289 (1991), 143-167.

[MSY] K. Morimoto, M. Sakuma and Y. Yokota, Identifying tunnel number one knots, to appear in J. Math. Soc. Japan.

[NR] W.D. Neumann and A.W. Reid, Arithmetic of hyperbolic manifolds, Topology 90 (ed. B. Apanasov et al.), (1992), pp. 273-310, Walter de Gruyter.

[P] C. Petronio, An algorithm producing hyperbolicity equations for a link complement in $S^{3}$, Geometry Dedicata, 44 (1992), 67-104.

[R1] R. Riley, Parabolic representations of knot groups, Proc. London Math. Soc., 24 (1972), 217-242.

[R2] R. Riley, A quadratic parabolic group, Math. Proc. Cambridge Phil. Soc., 77 (1975), 281-288.

[R3] R. Riley, An elliptic path from parabolic representations to hyperbolic structures, Lecture Notes in Math., 722 (1979), Springer-Verlag, pp. 99-133.

[R4] R. Riley, Seven excellent knots, Low-dimensional topology (ed. Brown and Thickstun), London Math. Soc. Lect. Note Series 48, pp. 81-151, Cambridge Univ. Press, 1982.

[Sa1] M. Sakuma, The geometries of spherical Montesinos links, Kobe J. Math., 7 (1990), $225-242$.

[Sa2] M. Sakuma, Minimal genus Seifert surfaces for special arborescent links, Osaka J. Math., 31 (1994), 861-905.

[SW] M. Sakuma and J. Weeks, The generalized tilt formula, Geometriae Dedicata, 50 (1995), $1-9$.

[Sc] H. Schubert, Knoten mit zwei Brücken, Math. Z., 65 (1956), 133-170.

[Si] L. Siebenmann, Exercices sur les noeuds rationnels, preprint. 
[Ta1] M. Takahashi, Two-bridge knots have Property $P$, Mem. Amer. Math. Soc., 29 (1981).

[Ta2] M. Takahashi, On the concrete construction of hyperbolic structure of 3-manifolds, Tsukuba J. Math., 9 (1985), 41-83.

[Th] W. Thurston, The geometry and the topology of 3-manifolds, mimeographed note.

[U] Y. Uchida, Detecting inequivalence of some unknotting tunnels for two-bridge knots, Proc. KAIST Math. Workshop 1990, pp. 227-232.

[We] J. Weeks, Convex hulls and isometries of cusped hyperbolic manifolds, Topology Appl., 52 (1993), 127-149.

[Wi] N.J. Wielenberg, Hyperbolic 3-manifolds which share a fundamental region, Ann. Math. Studies, 66, Princeton Univ. Press, pp. 505-513.

MAKOTO SAKUMA

Department of Mathematics

Graduate School of Science

OSAKA UNIVERSITY

MaChiKaneyama-CHO 1-16, TOYONAKA

OSAKA 560, JAPAN

e-mail: sakuma@math.wani.osaka-u.ac.jp

JEFFREY WEEKS

The Geometry Center

1300 South SECOND Street

MinNEAPOLIS MN 55454, USA

e-mail: weeks@geom.umn.edu 